\title{
COMPOSICIÓN FLORÍSTICA, DIVERSIDAD Y ECOLOGÍA DE HUMEDALES HERBÁCEOS EMERGENTES EN LA PLANICIE COStera Central de Veracruz, MÉXICO
}

\author{
Patricia Moreno-Casasola ${ }^{1,3}$, Eduardo Cejudo-Espinosa ${ }^{1}$, Ascención Capistrán-Barradas ${ }^{1}$, \\ Dulce Infante-Mata ${ }^{1}$, Hugo López-Rosas ${ }^{1}, 2$, Gonzalo Castillo-Campos ${ }^{1}$, Jesús Pale-Pale ${ }^{1}$ \\ y Adolfo Campos-Cascaredo ${ }^{1}$ \\ 1 Instituto de Ecología A.C. \\ ${ }^{2}$ Universidad del Mar \\ ${ }^{3}$ Autor para la correspondencia: patricia.moreno@inecol.edu.mx
}

\begin{abstract}
Resumen: Se describe la composición florística, diversidad y ecología de humedales herbáceos de la planicie costera de Veracruz, entre Tecolutla y la cuenca baja del río Papaloapan, teniendo éstos últimos mayor diversidad, principalmente laguna Conejo. Once localidades fueron muestreadas, encontrándose 95 especies (41 familias y 74 géneros). La clasificación jerárquica Beta Flexible conformó nueve grupos; para cada uno se graficó el régimen de inundación anual. La ordenación por componentes principales explicó el $47.81 \%$ de la variación mostrando un gradiente de riqueza de especies de los sitios de la zona norte y centro hacia los del Papaloapan, así como un gradiente de suelos Gleysol e Histosol (predominando histosoles en el Papaloapan). Existe gran variabilidad en la composición florística y dominancia de los humedales herbáceos emergentes y se considera que forman un complejo de riqueza de especies y combinaciones de monocotiledóneas y dicotiledóneas por lo que se sugiere usar el término popal-tular para denominarlos.
\end{abstract}

Palabras clave: Cyperus giganteus, diversidad, hidroperíodo, Sagittaria lancifolia, popal

\begin{abstract}
This work describes the floristic composition, ecology and diversity of marshes along the central coastal plain of Veracruz, from Tecolutla to the lowlands of the Papaloapan River, where more diverse sites were found (highest value in Conejo Lagoon). Eleven localities were sampled and 95 species were recorded (41 families and 74 genus). The classification (hierarchic Beta Flexible) grouped species in nine types and annual hydroperiods were represented graphically for each type. PCA explained $47.81 \%$ of the variation and samples showed a gradient in species richness from northern and central sites towards the Papaloapan as well as a soil gradient from Gleysols to Histosols (predominating in Papaloapan). The great variability in species composition and dominance suggests that they form a landscape complex with different combinations of monocotyledons and dicotyledons which should be named popal-tular.
\end{abstract}

Key words: Cyperus giganteus, diversity, freshwater marsh, hydroperiod, Sagittaria lancifolia.

L os humedales tienen gran importancia por los recursos que se obtienen de ellos (pesca, madera, miel, cacería, plantas medicinales, entre otros) y los servicios ambientales que proporcionan, ya que son la base de las actividades pesqueras, ayudan en el control de inundaciones, en la filtración y limpieza del agua y en la protección de las zonas costeras, en el aporte de nutrientes a los cuerpos de agua, proporcionan refugio a los juveniles de especies acuáticas entre los más importantes (Costanza et al., 1998; Semlitsch y Bodie, 1998). Conjuntan gran variedad de comunidades vegetales con distinta composición, estructura y formas de vida. Frecuentemente se les considera como un solo tipo de ecosistema, comparable a los bosques o pastizales, sin embargo, los humedales forman una enorme variedad de comunidades arbóreas y herbáceas (Wheeler et al., 2002). Los principales factores físicos involucrados en mantener la diversidad vegetal de los humedales son la microtopografía, el nivel y tiempo de inundación (Orozco-Segovia y Lot-Helgueras, 1976; Mitsch y Gosselink, 2000; FloresVerdugo et al., 2007), la salinidad en suelo y agua (Bedford et al., 1999; Moreno-Casasola et al., 2009). Estos factores varían en tiempo y espacio por lo que los humedales son 
ecosistemas altamente heterogéneos desde el punto de vista ambiental.

Se calcula que en el mundo existe aproximadamente una cobertura de 12.8 millones de $\mathrm{km}^{2}$ de humedales (Finlayson et al., 1999). Aunque todavía no se concluye el inventario nacional de humedales en México, el INEGI (2005) estima que cubren $0.66 \%$ de la superficie del país ( 1.22 millones de hectáreas), con cerca de 0.13 y 0.94 millones de hectáreas de popales y tulares. La pérdida de superficie de nuestros humedales debe de ser considerable ya que históricamente se han usado para la ganadería y la agricultura y más recientemente han sido sustituidos por las urbanizaciones, encontrándose actualmente entre los ecosistemas más amenazados (Moreno-Casasola et al., 2009).

Se considera que las mayores extensiones de humedales se localizan en las planicies costeras (Olmsted, 1993). Por ejemplo, en el Golfo de México están los complejos de humedales del Río Papaloapan y Pantanos de Centla, conformados por manglares, pastos marinos, selvas inundables, popales y tulares. Pero también existen humedales de menor extensión en la desembocaduras de los ríos Pánuco, Tecolutla y Palizada, en la planicie de inundación de las lagunas costeras y en los bordes de lagos de agua dulce (observación personal de los autores, Peralta-Peláez y Moreno-Casasola, 2009, entre otros).

Los humedales herbáceos emergentes incluyen a los popales, tulares y carrizales. Son ecosistemas ampliamente distribuidos en la planicie costera del Golfo de México. Los términos tular y popal fueron asignados por Faustino Miranda (Miranda y Hernández-X., 1963). El popal describe una comunidad vegetal sobre superficies pantanosas permanentemente estancada en la planicie costera de Tabasco, sur de Veracruz, Chiapas y Campeche. Se establece sobre llanuras aluviales, prácticamente sin declives, atravesadas por ríos que por medio de filtraciones e inundaciones cubren la superficie, en zonas con climas tropicales Af (Tropical húmedo con lluvias todo el año), Am (Tropical con lluvias monzónicas en verano) y Aw (Tropical sub-húmedo con lluvias en verano) (Rzedowski, 1983). Los tulares son comunidades de plantas acuáticas dominadas por monocotiledóneas de 1 a $3 \mathrm{~m}$ de alto, de hojas angostas o bien carentes de órganos foliares, entre el nivel del mar y los 2,750 m s.n.m., encontrándose en climas Af, Am, Aw, Cwa y Cwb (Templado subhúmedo con lluvias en invierno) (Rzedowski, 1983). Las comunidades formadas por Typha latifolia, T. domingensis, Scirpus spp., Cyperus giganteus, Cyperus spp. se conocen como tulares. En Veracruz, en la cuenca del Papaloapan, también se le nombra tule o tulillo a la ciperácea Eleocharis cellulosa. Los carrizales están dominados por Phragmites communis, Cladium jamaicense (Rzedowski, 1983) y Arundo donax (Martínez y Novelo, 1993). La diversidad de nombre locales y la mezcla de especies en una superficie determinada dificulta la aplicación de una terminología uniforme a los humedales herbáceos emergentes.
En México se han caracterizado floristícamente a los humedales herbáceos para varios sitios de la planicie costera del Golfo de México (Vázquez-Yañez, 1971; Orozco-Segovia, 1974; Orozco-Segovia y Lot-Helgueras, 1976; Rzedowski, 1983; Cházaro, 1986; Gutiérrez y Zolá, 1987; LotHelgueras y Novelo, 1988a; Lot-Helgueras, 1991; Martínez y Novelo, 1993; Olmsted, 1993; Ocaña y Lot-Helgueras, 1996; Lot-Helgueras, 2004; Novelo y Ramos, 2005; LópezRosas et al., 2005; Pérez et al., 2005; Moreno-Casasola et al., 2009; Peralta-Peláez y Moreno-Casasola, 2009). También se han estudiado las comunidades de herbáceas emergentes en las planicies costeras de la vertiente del Pacífico (Diego-Pérez et al., 1993; Fonseca y Lozada, 1993; DiegoPérez y Lozada-Pérez, 1994) y de los lagos de Michoacán (Lot y Novelo, 1988b; Rojas y Novelo, 1995). Estas zonas comparten algunas especies con los humedales herbáceos del Golfo de México como son Eichhornia crassipes, Salvinia minima, Typha domingensis, Thalia geniculata, Cyperus articulatus, Eleocharis spp. y Sagittaria latifolia.

Para las planicies inundables del sureste de Veracruz cercanas a Coatzacoalcos, Orozco-Segovia y Lot-Helgueras (1976) reportan asociaciones casi monoespecíficas de Eleocharis interstincta, de E. mutata, de Pontederia lanceolata, de Oryza latifolia, de Thalia geniculata, de Cyperus giganteus y de Typha domingensis, así como asociaciones de Typha domingensis- Cyperus giganteus, de Thalia geniculata-Pontederia lanceolata y de Pontederia lanceolata-Typha domingensis. En los humedales del Río Palizada, Campeche, Ocaña y Lot-Helgueras (1996) reportan la asociación de Typha domingensis-Eleocharis cellulosa, Leersia hexandra y Sagittaria lancifolia subsp. media, el carrizal de Phragmites australis, la comunidad de Typha-Phragmites, y el popal de Thalia-Pontederia-Sagittaria. Gutiérrez y Zolá (1987) para la zona de Neverías, en la cuenca baja del Río Actopan, reportan las agrupaciones de Cyperus articulatus, de Pontederia sagittata, de Hymenachne amplexicaulis, de Thalia geniculata, de Echinodorus andrieuxii, de Heliconia latispatha, de Limnocharis flava, de Xanthosoma robustum y de Eleocharis interstincta. Moreno-Casasola et al. (2009) reportan para la zona de la Mancha las agrupaciones de Thalia geniculata-Pontederia sagittata, la de Typha domingensis, la de Sagittaria lancifolia, la de Hydrocotyle bonariensis-Fimbristylis spadicea y la de Eleocharis mutata-Batis maritima. Peralta-Pelaéz y Moreno-Casasola (2009) registraron en las lagunas interdunarias de Veracruz las agrupaciones formadas por: i) Typha domingensis-Pontederia sagittata-Nymphoides indica-Salvinia auriculata, ii) Typha domingensis-Nymphoides indica-Eleocharis interstincta-Utricularia foliosa-Nymphaea ampla, iii) Pontederia sagittata-Hydrocotyle bonariensis- Eleocharis geniculata-Salvinia auriculata, y tres grupos con una presencia importante de gramíneas forrajeras: iv) Cynodon dactylon-Sagittaria lancifolia-Ipomoea carnea, v) Cyperus articulatus-Paspalum notatum- Hydrocotyle bonariensis, vi) Setaria geniculata-Thalia geniculata. 
Los humedales herbáceos emergentes se distribuyen desde el sur de Estados Unidos hasta Sudamérica. Se han publicado numerosos reportes sobre este tipo de humedales en Estados Unidos, específicamente en la costa este y en el delta del río Misisipi. En humedales del delta del Río Misisipi, se reportan comunidades de agua dulce dominadas por Sagittaria lancifolia, Panicum hemitomum, Zizaniopsis miliacea, Hydrocotyle spp., Pontederia cordata y Eleocharis spp. (Shiflet, 1963; Vissier et al., 1998). En los Everglades de Florida, los humedales herbáceos de agua dulce son dominados por Cladium jamaicense y Typha domingensis (Doren et al., 1997; Ewe et al., 2006). En Centroamérica, los humedales con influencia salobre o dulceacuícola presentan herbáceas emergentes como Sagittaria lancifolia, S. latifolia, Thalia geniculata, T. trichocalyx, Pontederia cordata, Phragmites australis, Typha angustifolia, T. domingensis, $T$. latifolia, así como varias especies de Cyperaceae y Poaceae (Ellison, 2004). Farruggia (2008) describe las sabanas inundables de Belice, con 33 especies de Poaceae y 27 de Cyperaceae. En Sudamérica, hay reportes de vegetación herbácea en zonas inundables estacionales o de desbordamiento de ríos en Brasil (Fortney et al., 2004) y en Argentina (Kandus y Adamoli, 1993; Padial et al., 2009).

Los humedales herbáceos están siendo transformados de manera importante en la planicie costera del Golfo de México. Se ha alterado su hidroperiodo al drenarlos o bien se han usado como potreros para ganado, introduciendo especies de pastos exóticos que toleran la inundación y van reemplazando a la vegetación nativa (Orozco-Segovia y Lot-Helgueras, 1976; Travieso-Bello et al., 2005; López Rosas, 2007). Estas actividades alteran el funcionamiento de los humedales, ya que además de desaparecer gran parte de la vegetación propia, el suelo pierde características como capacidad de filtración y retención de agua (López Rosas, 2007; Travieso-Bello et al., 2005). Como resultado de las modificaciones antrópicas o naturales, los humedales herbáceos registran cambios en el tiempo como respuesta a disturbios (Collins et al., 2001), pudiéndose identificar en ocasiones la causa de su alteración y el efecto resultante en sus comunidades vegetales. Este tipo de información es útil para evitar la degradación de los humedales existentes o tener éxito en su restauración.

El presente trabajo tiene como objetivo enriquecer el conocimiento de los humedales herbáceos emergentes de la planicie costera de Veracruz a través de la comparación de la composición florística y la estructura vegetal de once humedales herbáceos y relacionar su distribución con factores ambientales como es el hidroperíodo, el tipo de suelo y las características físico-químicas del agua.

\section{Materiales y Métodos}

Se ubicaron 11 localidades con presencia de humedales herbáceos emergentes (popales, tulares, ciperáceas o com- binaciones de éstos). En estas localidades se seleccionaron 13 humedales para el estudio de su vegetación y componentes abióticos (Figura 1 y Cuadro 1). Las localidades de La Mancha y de Río Blanco se dividieron en dos secciones que se analizaron (diversidad y parámetros físico-químicos) por separado debido a su composición florística contrastante. En La Mancha se separó la sección dominada por Typha domingensis de la dominada por Sagittaria lancifolia, denominados tular y popal respectivamente. En Río Blanco se separó la asociación Eleocharis cellulosa-Bacopa monnieri de la sección compuesta por Malvaviscus arboreus, Senna bicapsularis, Pithecellobium insigne y Spartina spartinae y se denominaron de la misma manera. En cada uno de los trece humedales se ubicaron unidades de muestreo en forma de cuadros de $2 \times 2 \mathrm{~m}$. De cada unidad de muestreo se obtuvo la cobertura total de la vegetación, así como la cobertura por especie usando la escala de cobertura-abundancia de Westhoff y van der Maarel (1978). Los ejemplares colectados se

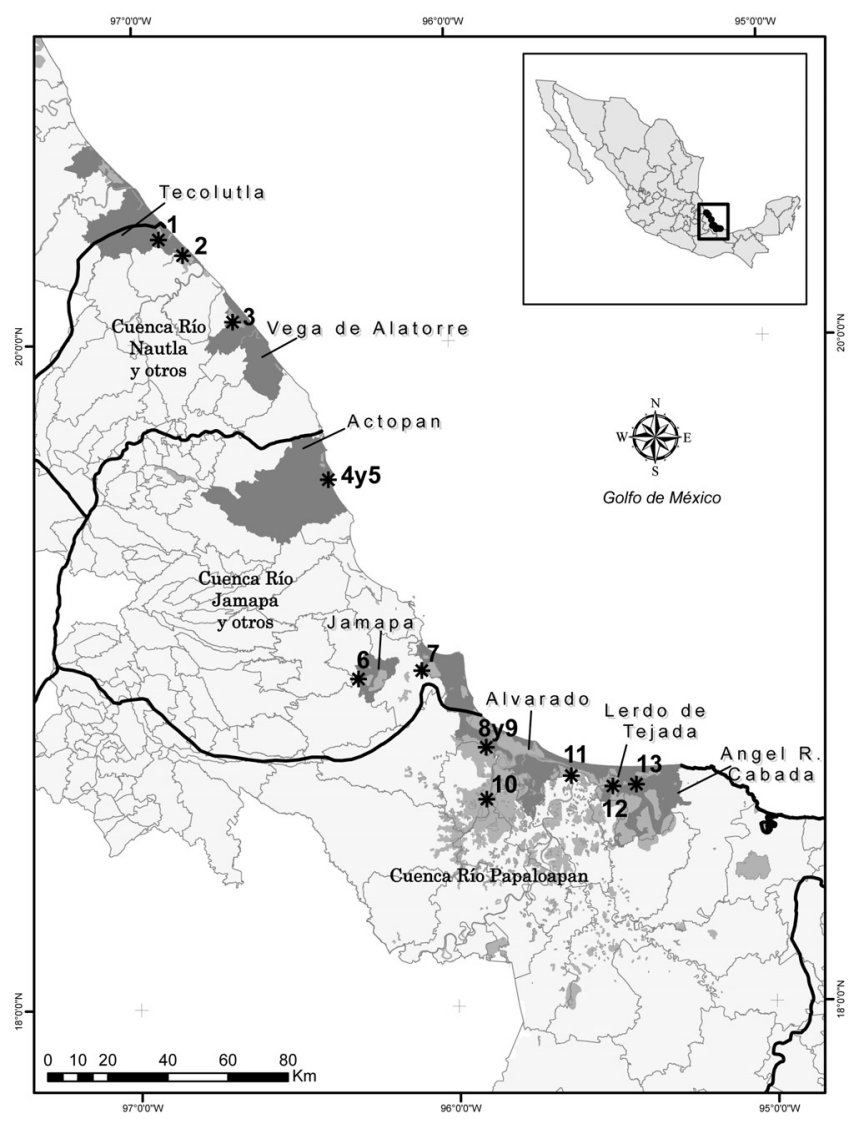

Figura 1. Mapa de localización de los humedales herbáceos estudiados en la planicie costera del Golfo de México en la zona centro del Estado de Veracruz, México. Nombre de humedales: 1) Ciénaga del Fuerte, 2) Estero Dulce, 3) Laguna Grande-Laguna Chica, 4) La Mancha tular, 5) La Mancha popal, 6) Laguna Apompal, 7) Laguna Mandinga, 8) Río Blanco popal, 9) Río Blanco tular, 10) Río Limón, 11) Laguna Conejo, 12) Sombrerete, 13) La Popotera. 
Moreno-Casasola et aL.

Cuadro 1. Localización y características de los sitios donde se muestrearon los humedales herbáceos. Precipitación anual histórica (1996-2003) en milímetros de acuerdo a CONAGUA (EriclII). El tipo de suelo se obtuvo de cada perfil edafológico (IUSS, 2006). Se presentan los valores promedio $\pm 1 \mathrm{EE}$ de la humedad relativa del suelo (Pw). Letras diferentes indican diferencias significativas entre las medias (ANOVA de una vía; $F=23.92, P<0.001)$. El clima se reporta según Soto (1986).

\begin{tabular}{|c|c|c|c|c|c|c|c|}
\hline $\begin{array}{l}\text { Nombre del } \\
\text { humedal }\end{array}$ & Municipio & Localización & Clima & $\begin{array}{l}\text { Precipitacion } \\
\text { anual }(\mathrm{mm})\end{array}$ & $\begin{array}{l}\text { Tipo de } \\
\text { suelo }\end{array}$ & $\begin{array}{l}\text { Pw (media } \pm \\
\text { e.e.) }\end{array}$ & Geoforma de paisaje \\
\hline Ciénaga del Fuerte & Tecolutla & $\begin{array}{l}20^{\circ} 18^{\prime} 49^{\prime \prime} \mathrm{N}, \\
96^{\circ} 55^{\prime} 22^{\prime \prime} \mathrm{W}\end{array}$ & $A m w "(e)$ & 1436.3 & $\begin{array}{l}\text { Gleysol } \\
\text { mólico }\end{array}$ & $375.9 \pm 22.1 \mathrm{a}$ & $\begin{array}{l}\text { Superficie lacuno-palustre } \\
\text { formada por depósitos } \\
\text { aluviales y biógenos }\end{array}$ \\
\hline Estero Dulce & Tecolutla & $\begin{array}{l}20^{\circ} 17^{\prime} 38^{\prime \prime} \mathrm{N}, \\
96^{\circ} 52^{\prime} 24^{\prime \prime} \mathrm{W}\end{array}$ & $A m w^{\prime \prime}(\mathrm{e})$ & 1436.3 & $\begin{array}{l}\text { Gleysol } \\
\text { mólico }\end{array}$ & $267.4 \pm 22.4$ b & $\begin{array}{l}\text { Superficie fluvio-acumulativa } \\
\text { formada por depósitos } \\
\text { biógenos y aluviales }\end{array}$ \\
\hline Laguna Grande y & Vega de & $20^{\circ} 05^{\prime} 46^{\prime \prime} \mathrm{N}$ & $\operatorname{Aw} 1\left(\mathrm{x}^{\prime}\right)(\mathrm{e})$ & 1397.7 & Gleysol & $211.9 \pm 16.7 \mathbf{c}$ & Superficie perilacustre \\
\hline Laguna Chica & Alatorre & $96^{\circ} 41^{\prime} 23^{\prime \prime} \mathrm{W}$ & & & hístico & & $\begin{array}{l}\text { acumulativa formada por } \\
\text { depósitos fluviales y biógenos }\end{array}$ \\
\hline La Mancha & Actopan & $\begin{array}{l}19^{\circ} 35^{\prime} 48^{\prime \prime} \mathrm{N}, \\
96^{\circ} 22^{\prime} 54^{\prime \prime} \mathrm{W}\end{array}$ & $A w 1 "(w)(i)$ & 1200.5 & $\begin{array}{l}\text { Histosol } \\
\text { sáprico }\end{array}$ & $68.9 \pm 9.7 \mathbf{f}$ & $\begin{array}{l}\text { Depositación intraduna } \\
\text { acumulativa formada por } \\
\text { depósitos marinos y biógenos }\end{array}$ \\
\hline Laguna La Apompal & Jamapa & $\begin{array}{l}19^{\circ} 01^{\prime} 23^{\prime \prime} \mathrm{N} \\
96^{\circ} 17^{\prime} 03^{\prime \prime} \mathrm{W}\end{array}$ & $\begin{array}{l}\text { Aw1" } \\
(w)(i) g\end{array}$ & 1591.5 & $\begin{array}{l}\text { Histosol } \\
\text { fíbrico }\end{array}$ & $288.5 \pm 38.2$ b & $\begin{array}{l}\text { Superficie palustre-fluvial } \\
\text { acumulativa formada por } \\
\text { depósito biógenos }\end{array}$ \\
\hline Laguna Mandinga & Alvarado & $\begin{array}{l}19^{\circ} 00^{\prime} 35^{\prime \prime} \mathrm{N} \\
96^{\circ} 05^{\prime} 33^{\prime \prime} \mathrm{W}\end{array}$ & $\begin{array}{l}\text { Aw2" } \\
(w)(e) g\end{array}$ & 1591.5 & $\begin{array}{l}\text { Gleysol } \\
\text { hístico }\end{array}$ & $289.1 \pm 36.4$ b & $\begin{array}{l}\text { Superficie lacustre } \\
\text { acumulativa formada por } \\
\text { depósitos lacustres y aluviales }\end{array}$ \\
\hline Río Blanco popal & Alvarado & $\begin{array}{l}18^{\circ} 44^{\prime} 47^{\prime \prime} \mathrm{N}, \\
95^{\circ} 52^{\prime} 42^{\prime \prime} \mathrm{W}\end{array}$ & $A w 2^{\prime \prime}(w)\left(i^{\prime}\right)$ & 1487.7 & $\begin{array}{c}\text { Histosol } \\
\text { sáprico sálico }\end{array}$ & $178.6 \pm 22.8 \mathbf{c}, \mathbf{d}, \mathbf{f}$ & $\begin{array}{l}\text { Superficie perilacustre } \\
\text { acumulativa formada por } \\
\text { depósitos lacustres y biógenos }\end{array}$ \\
\hline Río Blanco tular & Alvarado & $\begin{array}{l}18^{\circ} 44^{\prime} 47^{\prime \prime} \mathrm{N}, \\
95^{\circ} 52^{\prime} 42^{\prime \prime} \mathrm{W}\end{array}$ & $A w 2^{\prime \prime}(w)\left(i^{\prime}\right)$ & 1487.7 & $\begin{array}{c}\text { Gleysol hístico } \\
\text { endosálico }\end{array}$ & $165.7 \pm 16.9$ c,d,e & $\begin{array}{l}\text { Superficie perilacustre } \\
\text { acumulativa formada por } \\
\text { depósitos lacustres y biógenos }\end{array}$ \\
\hline Río Limón & Alvarado & $\begin{array}{l}18^{\circ} 38^{\prime} 10^{\prime \prime} \mathrm{N}, \\
95^{\circ} 53^{\prime} 47 \mathrm{~W}^{\prime \prime}\end{array}$ & $A w 2^{\prime \prime}(w)\left(i^{\prime}\right)$ & 1433.6 & Histosol fíbrico & $86.1 \pm 4.8 \mathbf{f}$ & $\begin{array}{l}\text { Superficie perilacustre } \\
\text { acumulativa formada por } \\
\text { depósitos lacustres y biógenos }\end{array}$ \\
\hline Laguna Conejo & Alvarado & $\begin{array}{l}18^{\circ} 42^{\prime} 22^{\prime \prime} \mathrm{N}, \\
95^{\circ} 37^{\prime} 32^{\prime \prime} \mathrm{W}\end{array}$ & $A w 2^{\prime \prime}(w)\left(i^{\prime}\right)$ & 1729.2 & Histosol tiónico & $112.7 \pm 4.4 \mathbf{e}, \mathbf{f}$ & $\begin{array}{l}\text { Superficie perilacustre } \\
\text { acumulativa formada por } \\
\text { depósitos lacustres y biógenos }\end{array}$ \\
\hline Sombrerete & Alvarado & $\begin{array}{l}18^{\circ} 39^{\prime} 48 \mathrm{~N}, \\
95^{\circ} 33^{\prime} 53 \mathrm{~W}\end{array}$ & Aw2"(e) & 2049.2 & Histosol fíbrico & $144.3 \pm 8.1 \mathbf{d}, \mathbf{e}$ & $\begin{array}{l}\text { Superficie perilacustre } \\
\text { acumulativa formada por } \\
\text { depósitos lacustres y biógenos }\end{array}$ \\
\hline Popotera & Lerdo & $\begin{array}{l}18^{\circ} 39^{\prime} 14^{\prime \prime} \mathrm{N}, \\
95^{\circ} 32^{\prime} 44 \mathrm{~W}^{\prime \prime}\end{array}$ & Aw2"(e) & 2049.2 & Histosol fíbrico & $114.7 \pm 8.5 \mathbf{e}, \mathbf{f}$ & $\begin{array}{l}\text { Superficie perilacustre } \\
\text { acumulativa formada por } \\
\text { depósitos lacustres y biógenos }\end{array}$ \\
\hline
\end{tabular}

determinaron y depositaron en el herbario del Instituto de Ecología A.C. (XAL). El listado aparece en el Apéndice 1.

En cada humedal se obtuvo el hidroperíodo en periodos bimestrales a lo largo de 16 meses (agosto 2007 a diciembre 2008). Los datos se obtuvieron de piezómetros (tubos de PVC de $1.3 \mathrm{~cm}$ de diámetro y $3 \mathrm{~m}$ de largo, enterrados a 1.5 $\mathrm{m}$ de profundidad, ranurados y cubiertos con malla de nylon en el extremo enterrado, para permitir el paso del agua, pero no de sedimentos (Peralta-Peláez et al., 2009) instalados previamente. En cada piezómetro se midió el nivel de agua con un sensor electrónico (marca Solinst $\left.{ }^{\circledR}\right)$. Junto con el nivel del agua, se obtuvieron las medidas de $\mathrm{pH}$, conducti- vidad y salinidad del agua intersticial a una profundidad de $30 \mathrm{~cm}$ usando un muestreador in situ de agua (McKee et al., 1988; Infante et al., 2009), utilizando un sensor multiparamétrico YSI modelo 556 MPS). Asimismo, de cada sitio se obtuvo una muestra de suelo que se pesó y posteriormente se secó a $60{ }^{\circ} \mathrm{C}$ por $72 \mathrm{~h}$ para obtener la humedad relativa $(\mathrm{Pw})$ con la fórmula (Peso húmedo - Peso seco)/ Peso seco $\times 100$ (Soil Science Laboratory Manual) y se empleó un Análisis de Varianza (Multiple Range Test, Statgraphics 5.1) para identificar diferencias en contenido de humedad del suelo superficial entre sitios. En cada sitio se excavaron perfiles edafológicos para identificar el tipo de suelo. 
Análisis de Datos. Se construyó una matriz con los datos de cobertura de las especies y otra con los datos de presencia/ausencia (ambas matrices de 95 especies, 104 cuadros) que se utilizaron para analizar la vegetación con métodos multivariados con el programa $\mathrm{PC}-\mathrm{Ord}(\mathrm{McCu}-$ ne y Mefford, 2006). Con base en los datos de cobertura, se obtuvo una clasificación numérica de las unidades de muestreo siguiendo la metodología Beta Flexible con un valor de $\beta=-0.25$, y como índice de similitud se utilizó la distancia Euclidiana relativizada, ya que conforme el valor de $\beta$ es más negativo, el método incrementa los espacios y los elementos se unen en grupos más compactos (McCune et al., 2002). Los datos de la matriz de presencia/ausencia fueron transformados con el Método de Beal's Smoothing (PC-Ord). Esta matriz se utilizó para hacer un Análisis de Componentes Principales $(A C P)$ y obtener la ordenación de las unidades de muestreo. Para calcular la diversidad alfa se utilizó el índice de Shannon y para la diversidad beta el índice de Sorensen. Para el cálculo de la diversidad beta se mantuvieron los sitios de La Mancha como uno solo debido a lo pequeño del sitio y a la cercanía entre ambas zonas de muestreo. El índice de Shannon se calculó usando la fórmula

$\mathrm{H}^{\prime}=-\sum \mathrm{p}_{\mathrm{i}} \log \mathrm{p}_{\mathrm{i}}$

donde $\mathrm{p}_{\mathrm{i}}=$ probabilidad de importancia en la columna $\mathrm{i}=$ matriz de elementos relativizada por el total de filas (GreigSmith, 1983 basado en Shannon y Weaver, 1949). Se calculó el valor de importancia relativa $(V I R)$ por especie con la fórmula $V I R=[(F R+C R) / 2] \times 100$, donde la frecuencia relativa $(F R)$ se definió como el número de cuadros donde aparece la especie, dividido entre el número total de cuadros muestreados. La cobertura relativa $(C R)$ se consideró como la cobertura promedio de la especie en los cuadros donde aparece (según escala de cobertura abundancia), dividido entre la cobertura total del cuadro para todas las especies.

Los datos físico-químicos del agua intersticial de cada humedal se compararon con un análisis de varianza de una vía (Zar, 1984). Cuando se detectaron diferencias significativas entre medias con $\mathrm{p}<0.05$, se aplicó una prueba de Tukey para grupos con número de muestras diferentes, empleando el programa Statistica Version 5.1 (StatSoft, 1998). Los valores de $\mathrm{Pw}$ del suelo de cada sitio se compararon con un análisis de varianza no paramétrico (Multiple Range Test, Statgraphics 5.1).

De cada uno de las agrupaciones florísticas generadas en el análisis de clasificación jerárquica se obtuvo una gráfica de hidroperiodo.

\section{Resultados}

Se colectaron 95 especies, pertenecientes a 74 géneros y 41 familias, con siete especies sin identificar. En el Apéndice 1 se presenta la lista de especies, su forma de crecimiento y su valor de importancia por sitio y global. Se registraron 34 especies de monocotiledóneas, 58 de dicotiledóneas y tres helechos.

Solamente veintidós especies aparecieron en más de 10 cuadros. En orden decreciente de frecuencia están Typha domingensis (en 45 cuadros), Thalia geniculata (24), Cyperus giganteus (22), Echinochloa pyramidalis (20), Cyperus articulatus y Pontederia sagittata (17), Salvia sp. (16), Sagittaria lancifolia subsp. media (15), Leersia ligularis (14), Sagittaria lancifolia (14), Vigna luteola (14), Eleocharis cellulosa (13), Hydrocotyle bonariensis y Zanthoxylum
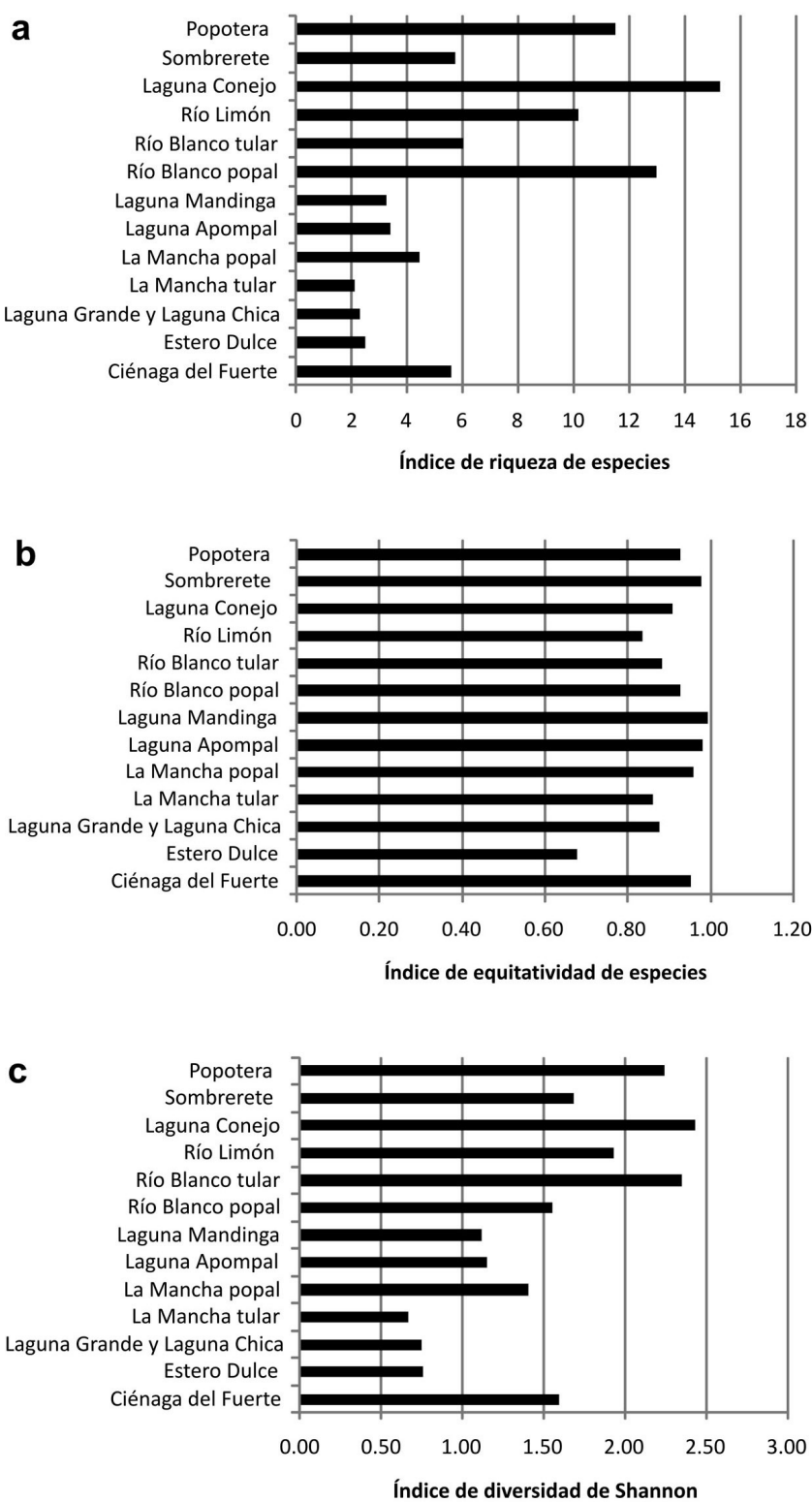

Figura 2. Gráficas que permiten comparar (a) valores de riqueza, (b) equitatividad y (c) diversidad (mediante el índice de Shannon) de los trece humedales herbáceos muestreados. 
caribaeum (12), Dalbergia brownei, Leersia sp. y Zizaniopsis miliacea (11), y Bacopa monnieri, Funastrum clausum, Hymenachne amplexicaulis, Scleria sp. y Sesbania sp., presentes en 10 cuadros. Treinta y dos especies aparecieron en menos de tres cuadros.

Los mayores valores de importancia relativa se encontraron en Typha domingensis (12.31), Echinochloa pyramidalis (10.33), Thalia geniculata (7.97), Cyperus giganteus (7.34), Leersia ligularis var. ligularis (6.33), Pontederia sagittata (5.83), Sagittaria lancifolia subsp. media (5.12), Sagittaria lancifolia (4.98), Cyperus articulatus (4.49), Zizaniopsis miliacea (4.11). Las familias con mayor número de especies con valores de importancia altos en orden decreciente son las Poaceae, Cyperaceae, Alismataceae y Fabaceae. Los valores por especie pueden verse en el Apéndice 1.

La riqueza de especies por sitios es variable entre humedales. En la figura 2 se observan los valores de riqueza de especies (a), la equitatividad (b) y la diversidad usando el índice de Shannon (c). La mayor riqueza de especies se encontró en los humedales de Laguna Conejo (15.29) y el humedal identificado como Río Blanco popal (13.0), seguido por la Popotera (11.5) y Río Limón (10.17), todos ellos ubicados en el Papaloapan. La misma tendencia se presenta en los valores de diversidad alfa: Laguna Conejo (2.44), Río Blanco popal (2.35), Popotera (2.25), Río Limón (1.93), Sombrerete (1.69) y Ciénaga del Fuerte (1.60). Los valores más bajos se encontraron en Estero Dulce y en Laguna Grande-Laguna Chica (0.76). Por tanto, los valores más altos corresponden a las comunidades herbáceas ubicadas en los humedales del bajo Papaloapan y en Ciénaga del Fuerte, en la planicie de inundación del Río Tecolutla. La mayor equitatividad se encontró en los humedales de Mandinga, Apompal, Sombrere- te y Ciénaga del Fuerte. En este caso, los humedales del bajo Papaloapan se ubican en los valores intermedios.

La diversidad beta (Cuadro 2) mostró que las comunidades de los distintos sitios difieren considerablemente entre sí. Lo valores de similitud más altos se registraron entre los humedales de Estero Dulce y Laguna Grande, entre Laguna Apompal y Laguna Mandinga y entre Río Blanco tular y Mandingna, Apompal y Laguna Conejo. En el otro extremo, con cero similitud, están Ciénaga del Fuerte con varios otros humedales (Mandinga, Apompal, Río Limón, Río Blanco tular). Lo mismo se encontró entre Estero Dulce y los humedales de Río Limón, de Río Blanco tular y Conejo, así como entre Río Blanco popal y Laguna Grande y Chica. Laguna Apompal también presentó valor de similitud de cero con La Popotera y Mandinga.

Los parámetros medidos en el agua intersticial entre sitios también presentaron diferencias. En el cuadro 3 se observa el resumen de los análisis de varianza para los parámetros de conductividad, salinidad, $\mathrm{pH}$ y nivel de inundación. La figura 3 muestra los valores de la salinidad, la conductividad, el pH y el nivel máximo de inundación y su comparación estadística para los 13 sitios muestreados. La conductividad del agua intersticial mostró un intervalo de 0.49 a $12.06 \mathrm{mS} \mathrm{cm}^{-1}$ (Figura 3a). Los humedales que presentaron valores menores a $2 \mathrm{mS} \mathrm{cm}^{-1}$ fueron Ciénaga del Fuerte, La Mancha popal, Laguna Apompal, Laguna Grande-Laguna Chica y La Popotera. Con valores mayores a $6 \mathrm{mS} \mathrm{cm}^{-1} \mathrm{se}$ encuentran Río Blanco tular y popal y Laguna de Mandinga. Los valores más altos de conductividad se localizan en los humedales del Papaloapan, a excepción de La Popotera. La salinidad presentó un comportamiento similar, fluctuando entre 0.25 y 7.54 partes por mil (Figura 3b).

Cuadro 2. Diversidad beta que muestra las similitudes entre los humedales herbáceos emergentes de los sitios muestreados. La Mancha popal y tular se mantuvieron como un solo sitio debido a su cercanía y a la superficie pequeña que ocupan. Se agregó la localidad de Coatzacoalcos y los alrededores del sureste de Veracruz, utilizando la lista florística presentada por Orozco-Segovia y Lot-Helgueras (1976). A pesar de que acumulan las especies encontradas en varios sitios de muestreo de la zona, presentan valores bajos de similitud con el resto de las zonas de trabajo. En negritas se indican los valores altos de similitud y en subrayado los valores bajos.

\begin{tabular}{|c|c|c|c|c|c|c|c|c|c|c|c|c|c|}
\hline & $\begin{array}{l}\text { L.Grande- } \\
\text { Chica }\end{array}$ & Ciénaga & L.Mandinga & E.Dulce & L.Apompal & $\begin{array}{c}\text { R.Blanco } \\
\text { tular }\end{array}$ & R.Limón & $\begin{array}{c}\text { R.Blanco } \\
\text { popal }\end{array}$ & Popotera & Sombrerete & L.Conejo & La Mancha & Coatzacoalcos \\
\hline L.Grande-Chica & & 0.2 & 0.14 & 0.4 & 0.25 & 0.24 & 0.15 & 0 & 0.18 & 0.22 & $\underline{0.07}$ & 0.17 & 0.1 \\
\hline Ciénaga & & & $\underline{0}$ & 0.11 & $\underline{0.08}$ & 0 & $\underline{0}$ & $\underline{0.07}$ & $\underline{0.07}$ & 0.15 & $\underline{0.05}$ & $\underline{0.06}$ & $\underline{0.03}$ \\
\hline L.Mandinga & & & & 0.17 & 0.44 & 0.42 & 0.21 & $\underline{0.08}$ & 0 & 0.1 & 0.25 & 0.16 & 0.13 \\
\hline E.Dulce & & & & & 0.14 & $\underline{0}$ & $\underline{0}$ & 0.1 & 0.2 & 0.25 & 0 & 0.19 & $\underline{0.07}$ \\
\hline L.Apompal & & & & & & 0.38 & 0.19 & 0 & $\underline{0.08}$ & 0.18 & 0.18 & $\underline{0.07}$ & $\underline{0.09}$ \\
\hline R.Blanco tular & & & & & & & 0.38 & 0.15 & $\underline{0.07}$ & 0.17 & 0.46 & 0.14 & 0.15 \\
\hline R.Limón & & & & & & & & 0.22 & 0.22 & 0.24 & 0.27 & 0.16 & $\underline{0.08}$ \\
\hline R.Blanco popal & & & & & & & & & 0.19 & 0.14 & $\underline{0.2}$ & $\underline{0.06}$ & $\underline{0.03}$ \\
\hline Popotera & & & & & & & & & & 0.5 & $\underline{0.05}$ & 0.18 & $\underline{0.06}$ \\
\hline Sombrerete & & & & & & & & & & & 0.22 & 0.14 & 0.12 \\
\hline L.Conejo & & & & & & & & & & & & 0.24 & 0.1 \\
\hline La Mancha & & & & & & & & & & & & & $\underline{0.08}$ \\
\hline Coatzacoalcos & & & & & & & & & & & & & \\
\hline
\end{tabular}



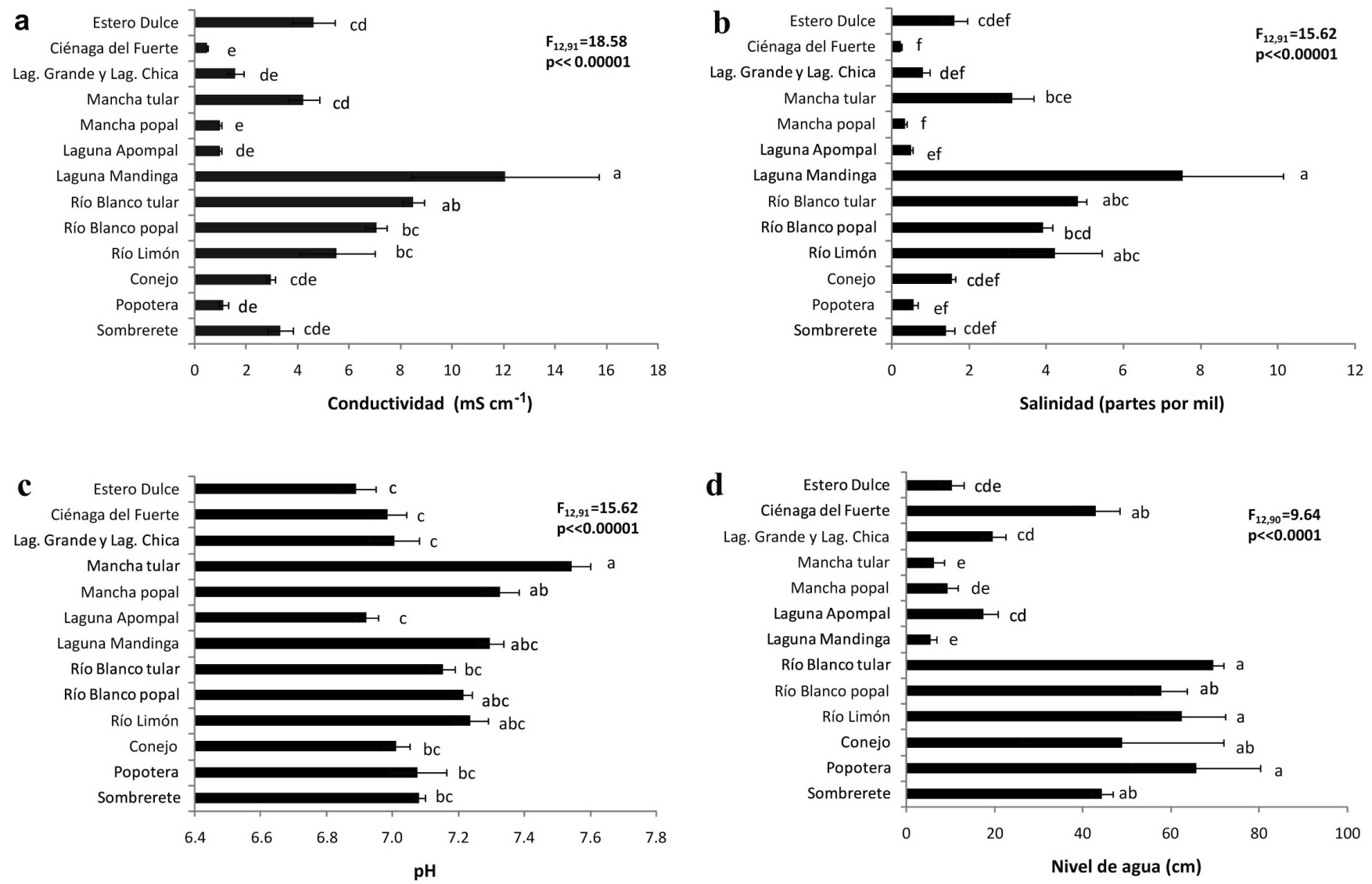

Figura 3. Conductividad (a), salinidad (b), pH (c) del agua intersticial y nivel de inundación (d) de trece humedales herbáceos de la planicie costera de Veracruz. Letras diferentes indican diferencias significativas, $\mathrm{p}<<0.00001$.

Los humedales herbáceos presentaron un $\mathrm{pH}$ entre 6.8 y 7.5 , es decir que hay muy poca variación entre ellos a pesar de presentarse diferencias significativas en los valores. Los humedales que tuvieron un $\mathrm{pH}$ menor a 7 son Estero Dulce, Laguna Apompal y Ciénaga del Fuerte. El único humedal que presentó un valor de 7.5 fue La Mancha tular (Figura 3c). Cabe decir que los humedales del Papaloapan se ubica-

Cuadro 3. Análisis de varianza para parámetros de conductividad, salinidad, pH e inundación. ${ }^{* * *} \mathrm{p}<0.0001$.

\begin{tabular}{lccccc}
\hline & $\begin{array}{c}\text { Grados de } \\
\text { libertad }\end{array}$ & $\begin{array}{c}\text { Suma de } \\
\text { cuadrados }\end{array}$ & $\begin{array}{c}\text { Grados de } \\
\text { libertad } \\
\text { del error }\end{array}$ & $\begin{array}{c}\text { Suma de } \\
\text { cuadrados } \\
\text { del error }\end{array}$ & $\mathrm{F}$ \\
\hline $\begin{array}{l}\text { Conductividad } \\
\text { (mS/cm) }\end{array}$ & 12 & 71.48 & 91 & 3.85 & $18.59^{* * *}$ \\
$\begin{array}{l}\text { Salinidad } \\
\text { (partes por mil) }\end{array}$ & 12 & 29.69 & 91 & 1.90 & $15.63^{* * *}$ \\
pH & 12 & 0.34 & 91 & 0.04 & $9.65^{* * *}$ \\
$\begin{array}{l}\text { Nivel de inun- } \\
\text { dación (cm) }\end{array}$ & 12 & 4505.80 & 90 & 428.91 & $10.51^{* * *}$ \\
\hline
\end{tabular}

ron en los valores centrales, por debajo de La Mancha.

El nivel de inundación de los humedales fluctuó entre 5.5 y $69.5 \mathrm{~cm}$ sobre el nivel del suelo. Los humedales con menos de $20 \mathrm{~cm}$ de inundación fueron los ubicados en el centro-norte del Estado e incluyen Laguna Mandinga, La Mancha tular y popal, Estero Dulce, Laguna Apompal y Laguna Grande-Laguna Chica. El resto de los humedales presentó un nivel de agua mayor a $43 \mathrm{~cm}$ y se ubicaron en el Papaloapan, con excepción de Ciénaga del Fuerte en Tecolutla. La figura $3 \mathrm{~d}$ muestra los valores de inundación y los análisis estadísticos indican diferencias significativas entre los humedales.

La clasificación jerárquica $\beta$ Flexible se presenta en la figura 4 , con un valor de encadenamiento de 0.97 , formándose nueve grupos. La primera gran división separa a los tres grupos con dominancia de Typha domingensis, Zizaniopsis miliacea, Zanthoxylum caribaeum y Sagittaria lancifolia subsp. media (grupo I- 36 cuadros de muestreo) de otros grupos (grupo II) En éste se separan dos grandes conjuntos, por un lado las agrupaciones donde predomina Cyperus giganteus y Thalia geniculata (grupo 4), y por el otro agrupaciones con menor número de muestras, pero una mayor 


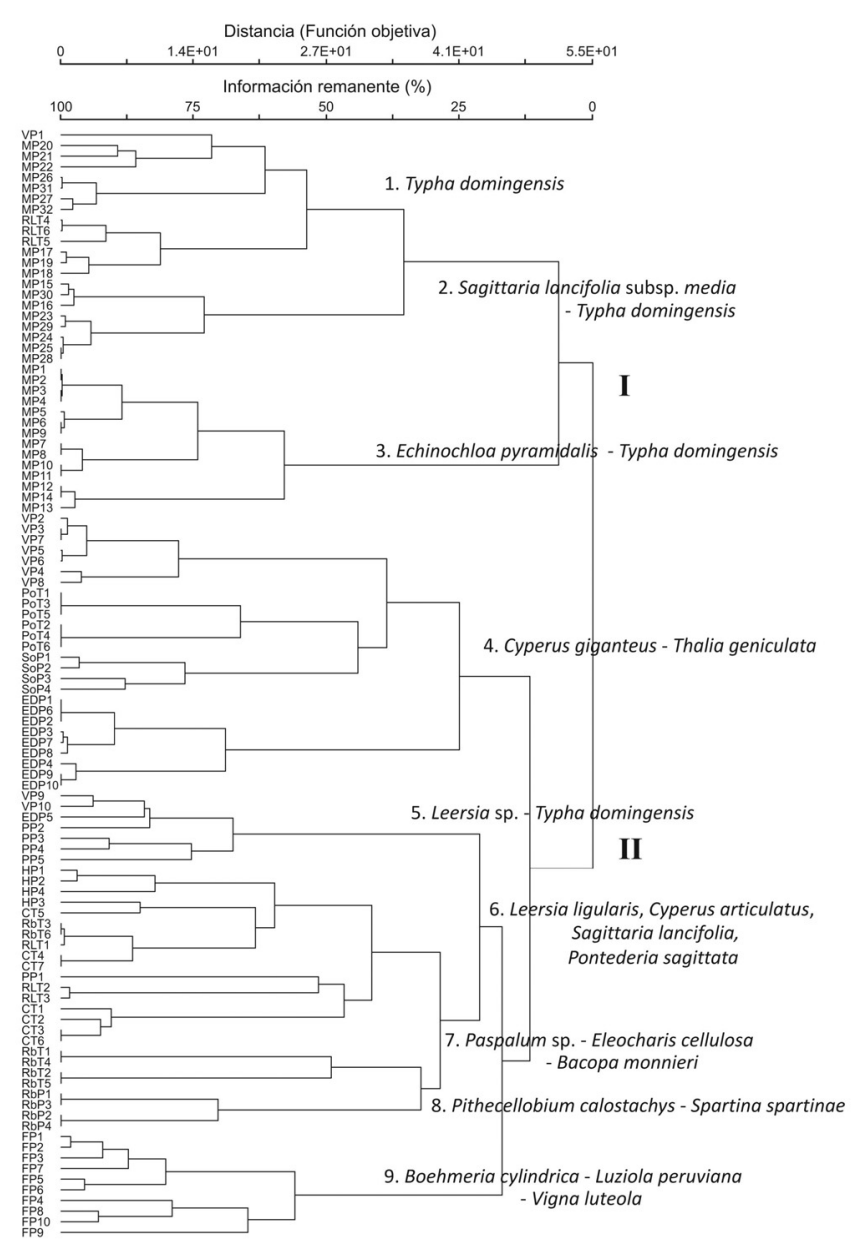

Figura 4. Dendrograma de las muestras de trece popales-tulares utilizando la clasificación Beta Flexible y la distancia euclidiana relativa como medida de similitud, con un encadenamiento de 0.97 . En la figura se indican las especies más frecuentes y con mayores valores de cobertura en cada uno de los nueve grupos.

variedad de especies (grupos 5 a 9). En el Apéndice 2 se describe cada una de las agrupaciones.

Los hidroperiodos (Figura 5) muestran las curvas representativas de la fluctuación del agua a lo largo del año. En la figura mencionada (5a) aparece un conjunto de dos hidroperiodos que corresponden a las agrupaciones florísticas 2 (Sagittaria lancifolia subsp. media- Typha domingensis) y 5 (Leersia sp. -Typha domingensis). Son las que pasan mayor tiempo inundadas. El segundo grupo (Figura 5 b) conjunta los hidroperiodos en los que el agua se mantiene por encima del suelo durante varios meses del año, aunque durante la temporada de secas se reduce el nivel del agua. Corresponde a las agrupaciones florísticas 1 (Typha domingensisZanthoxylum caribaeum-Zizaniopsis miliacea), 4 (Cyperus giganteus-Thalia geniculata), 7 (Paspalum sp.-Eleocharis cellulosa-Bacopa monnieri) y la 8 (Pithecellobium calostachys-Spartina spartinae). El tercer conjunto está formado por los hidroperiodos de las agrupaciones de Echinochloa
pyramidalis-Typha domingensis (3), de Boehmeria cylindrica-Luziola peruviana-Vigna luteola (9) y de Leersia ligularis-Cyperus articulatus (6). Son los grupos que permanecen inundados menor tiempo. Como puede verse en la figura $5 \mathrm{c}$, una buena parte del año el agua se encuentra por debajo del nivel del suelo.

La ordenación por componentes principales (Figura 6) explica el $47.810 \%$ de la varianza acumulada entre los dos primeros ejes. En el eje 1 hacia el lado izquierdo del espacio de ordenación se separan los cuadros de vegetación de los humedales del Papaloapan (Conejo, Limón, Río Blanco, Sombrerete- aunque la Popotera tiende hacia la derecha) y Mandinga, en los que dominan Pontederia sagittata, Sagittaria lancifolia, Cyperus articulatus, Typha domingensis, Eleocharis cellulosa, Zizaniopsis miliacea. Hacia la derecha y tendiendo hacia la parte superior se ubican los humedales de la zona centro norte del Estado, entre los que La Mancha y Laguna Apompal se ubican hacia el centro del espacio de ordenación. Como ya se mencionó, los humedales del Papaloapan son los más diversos por lo que también se puede interpretar un gradiente de riqueza de especies, con los valores más bajos arriba y a la derecha (excepto algunas muestras de Ciénaga del Fuerte con siete a nueve especies en la parte superior) y los mayores valores en la parte inferior y a la izquierda.

Todos los humedales herbáceos muestreados se establecen sobre variantes de dos tipos de suelo: Gleysol (Ciénaga del Fuerte, Laguna Grande-Laguna Chica, Estero Dulce, Mandinga, Río Blanco tular) e Histosol (Apompal, así como los humedales del Papaloapan- Río Blanco popal, Río Limón, Conejo, Sombrerete, Popotera). El eje uno de la ordenación se puede interpretar también como un gradiente edáfico ya que en la parte superior y a la derecha predominan los sitios con suelos tipo Gleysol y en la parte inferior y a la izquierda aquellos con suelos tipo Histosol, aunque las muestras de Río Blanco popal y Mandinga quedan mezcladas con los sitios de Histosoles. El análisis realizado al promedio anual del contenido de humedad en el suelo superficial indica que hay diferencias entre los sitios muestreados (Cuadro 1) siendo mayor en Ciénaga del Fuerte $(\mathrm{Pw}=375.9)$, los intermedios entre 200 y 300 se ubican en la zona central (correspondiente a los Gleysoles), las muestras con valores entre 100 y 199 en la parte inferior, aunque los valores menores (50 a 100, en los suelos del tipo Histosol de La Mancha y Río Limón), se ubican hacia la parte central, derecha, oscureciendo este gradiente.

Las especies con cargas negativas sobre el eje 1 fueron Cyperus articulatus $(r=-0.3328)$, Leersia ligularis $(r=$ 0.3125), Eleocharis cellulosa ( $r=-0.2983)$, Hydrocotyle bonariensis $(r=0.2827)$, Pontederia sagittata $(r=-0.2741)$ y Bacopa monnieri $(r=-0.2151)$. Con valores positivos las especies con mayores cargas son Thalia geniculata $(r=$ 0.22), Cyperus giganteus ( $r=0.1965)$, Echinochloa pyramidalis $(r=0.1133)$, Hymenachne amplexicaulis $(r=0.1044)$, 
Typha domingensis $(r=0.099)$. Sobre el segundo eje las especies con carga positiva localizadas en la parte superior de la gráfica fueron Boehmeria cylindrica $(r=0.1973)$, Salvia $s p .(r=0.1767)$, Luziola peruviana $(r=0.154)$, Ipomoea $s p$. $(r=0.1299)$ y Sagittaria lancifolia subsp. media $(r=0.129)$, mientras que con valores negativos aparecen Mimosa pigra $(r=-0.2538)$, Thalia geniculata $(r=-0.265)$, Ipomoea squamosa $(r=-0.2668)$, Cyperus giganteus $(r=-0.2873)$, Hymenachne amplexicaulis $(r=-0.2967)$.

\section{Discusión}

La información recabada muestra que los humedales herbáceos de la planicie costera central de Veracruz no son homogéneos en su estructura y composición de especies. Existen especies ampliamente distribuidas y dominantes, pero otras que alcanzan valores altos de cobertura y frecuencia, contribuyen a la diversidad de estas comunidades. En el presente trabajo registramos 95 especies para los humedales herbáceos muestreados, en aproximadamente $300 \mathrm{~km}$ de la planicie costera del Golfo de México, de las cuales 11 especies son reportadas como frecuentes en Centroamérica (Ellison, 2004). Las monocotiledóneas resultaron ser las de mayor VIR en los humedales emergentes estudiados (Apéndice 1). Si se analiza no solo la presencia, sino también un valor cuantitativo como la cobertura, siete especies tienen valores por arriba del $50 \%$ de cobertura por cuadro: Cyperus giganteus, Thalia geniculata, Typha domingensis, Pontederia sagittata, Leersia ligularis, Scleria sp. y Cyperus articulatus.

El valor de importancia alto de algunas especies obedece a dos razones. La primera es que tienen valores relativamente altos de cobertura y frecuencia en más de cuatro sitios (este número de sitios fue definido arbitrariamente y representa la tercera parte de las zonas de muestreo) siendo éste el caso de Sagittaria lancifolia, Cyperus articulatus, Cyperus giganteus, Eleocharis cellulosa, Scleria sp., Thalia geniculata, Pontederia sagittata, Typha domingensis, Vigna luteola. Ello habla de una clara dominancia de una o pocas especies. La segunda razón son valores altos de cobertura en pocos sitios (1 a 3), en donde son especies dominantes Sagittaria lancifolia subsp. media, Echinochloa pyramidalis, Hymenachne amplexicaulis, Leersia ligularis, Leersia sp., Zizaniopsis miliacea, Ipomoea squamosa, Dalbergia brownei, Salvia sp., Zanthoxylum caribaeum, Boehmeria cylindrica.

Las comunidades de humedales herbáceos emergentes reportadas tanto en la literatura como en el presente trabajo, reúnen diversas agrupaciones florísticas. Hay especies ampliamente distribuidas que constituyen comunidades donde dominan por si solas o en combinación con otras especies: Typha domingensis, Thalia geniculata, Pontederia sagittata, Cyperus giganteus, Phragmites communis y en menor grado Cyperus articulatus, Sagittaria lancifolia. Algunas otras aparecen como especies asociadas y solo en algunos sitios adquieren dominancia como en el caso de Oryza la-
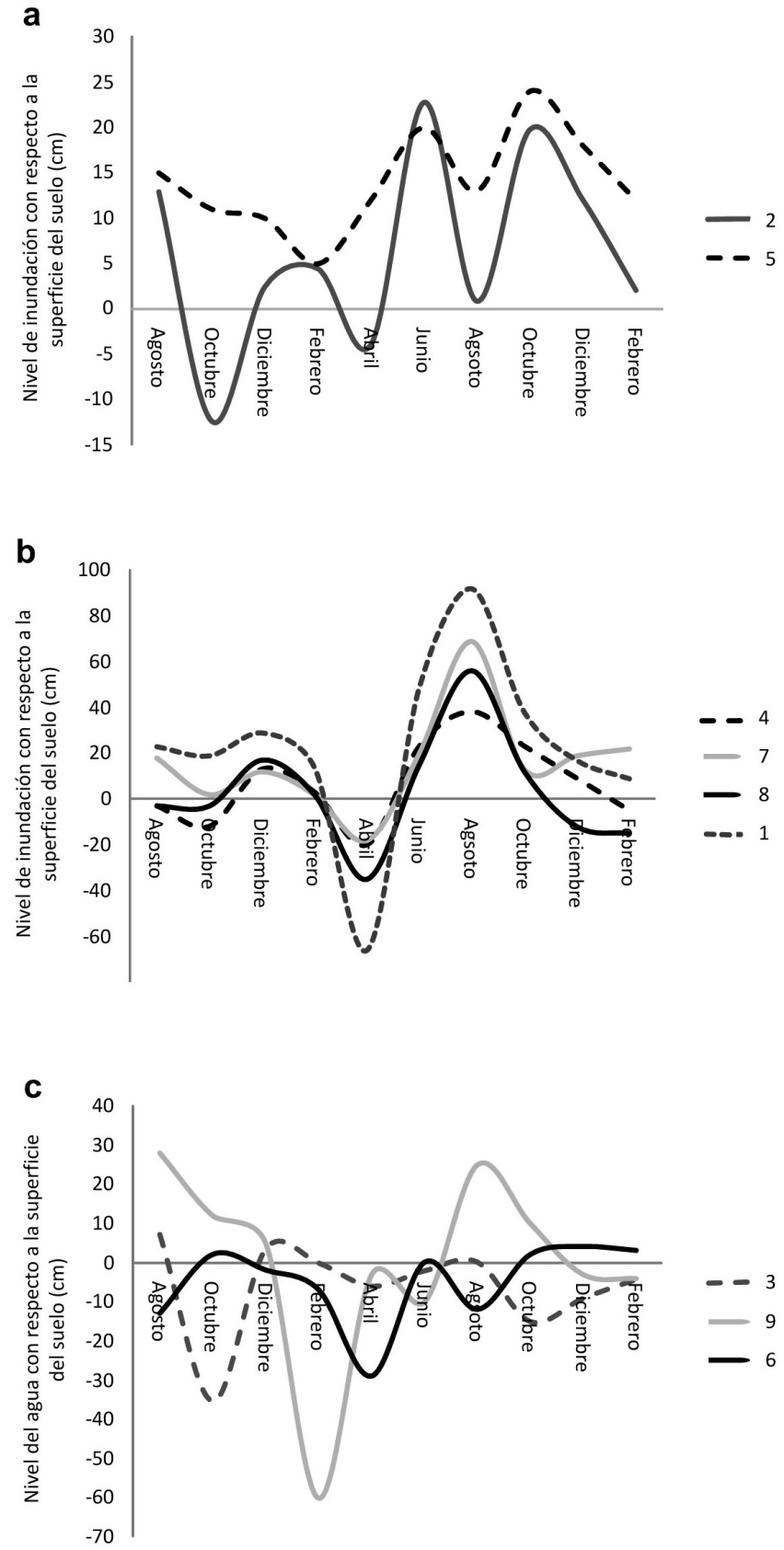

Figura 5. Hidroperíodo anual de humedales herbáceos. a) corresponden a las agrupaciones florísticas 2 (Sagittaria lancifolia subsp. media- Typha domingensis) y 5 (Leersia sp. -Typha domingensis), que pasan mayor tiempo inundadas. b) en ellos el agua se mantiene por encima del suelo durante varios meses del año reduciéndose en la temporada de secas y corresponden a las agrupaciones florísticas 1 (Typha domingensis- Zanthoxylum caribaeum- Zizaniopsis miliacea), 4 (Cyperus giganteus-Thalia geniculata), 7 (Paspalum sp.-Eleocharis cellulosa-Bacopa monnieri) y 8 (Pithecellobium calostachys- Spartina spartinae). c) corresponde a la agrupación 3 (Echinochloa pyramidalis-Typha domingensis), 9 (Boehmeria cylindrica- Luziola peruviana-Vigna luteola) y 6 (Leersia ligularis-Cyperus articulatus), las cuales permanecen inundadas menor tiempo. 


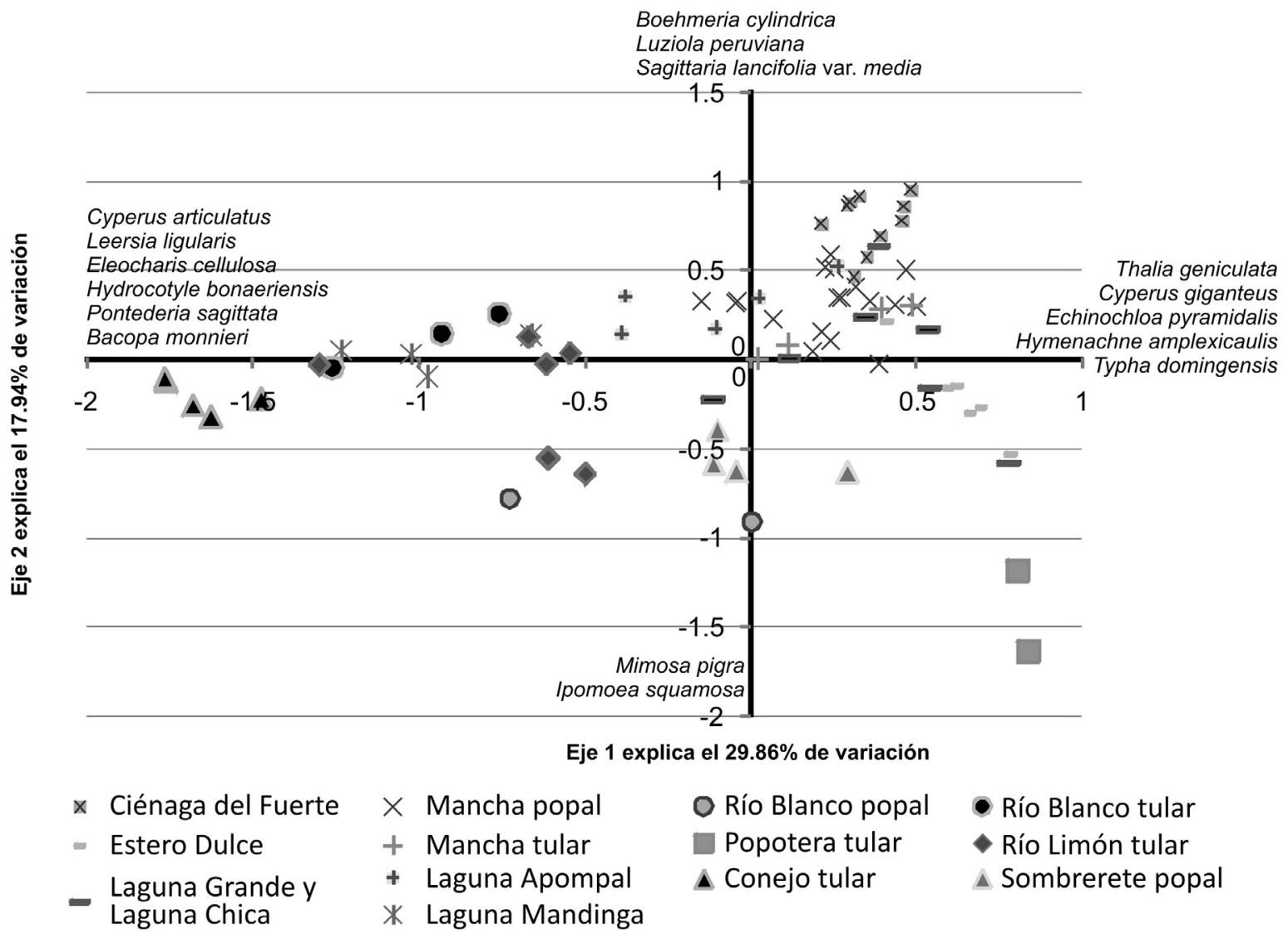

Figura 6. Ordenación mediante un Análisis de Componentes Principales (transformación de datos con Beal's Smoothing) de muestras de trece humedales herbáceos. Se explica el $48.81 \%$ de la varianza acumulada. Se interpretó como un gradiente de riqueza de especies sobre el eje 1, separándose los cuadros de vegetación de los humedales del Papaloapan, los de mayor riqueza (Conejo, Limón, Río Blanco, Sombrerete- aunque la Popotera tiende hacia la derecha) y Mandinga. El eje uno de la ordenación se puede interpretar también como un gradiente edáfico ya que en la parte superior y a la derecha predominan los sitios con suelos tipo gleysol y en la parte inferior y a la izquierda aquellos con suelos tipo histosol, aunque las muestras de Río Blanco popal y Mandinga quedan mezcladas con los sitios de histosoles.

tifolia, Eleocharis interstincta, Eleocharis mutata, Pontederia lanceolata, Eleocharis cellulosa, Leersia hexandra, Hymenachne amplexicaulis, Echinodorus andrieuxii, Heliconia latispatha, Limnocharis flava, Xanthosoma robustum, Sagittaria lancifolia subsp. media, Eleocharis cellulosaBacopa monnieri, Luziola-Setaria-Vigna luteola, Spartina spartinae, Eleocharis mutata-Batis maritima, Hydrocotyle bonariensis- Fimbristylis spadicea, Eleocharis interstincta- Nymphoides indica, Eleocharis geniculata con Hydrocotyle bonariensis y Pontederia sagittata (Orozco-Segovia y Lot-Helgueras, 1976; Gutiérrez y Zolá, 1987; Ocaña y Lot-Helgueras, 1996; Moreno-Casasola et al., 2009; Peralta y Moreno-Casasola, 2009).

Typha domingensis es la especie con mayor frecuencia y es importante mencionar que se pueden encontrar tulares monoespecíficos dominados por esta especie. Es una de las primeras en colonizar una zona que recién mantiene un nivel de inundación de varios meses, por ejemplo a orilla de carreteras, y su capacidad de expansión le permite ocupar el área y dificultar la entrada de otras especies. También se ha reportado que es una especie que se ve favorecida por el incremento en nutrientes (Woo y Zedeler, 2002; Lorenzen et $a l ., 2001)$ y hoy en día, las planicies costeras reciben el escurrimiento de los agroquímicos de muchos cultivos, siendo la eutroficación de las aguas uno de los principales problemas ambientales en cuerpos de agua interiores y costeros. En los humedales bajo restauración de La Mancha se ha visto como se está incrementado su presencia (López-Rosas et al., 2010).

Los valores de riqueza de especies, equitatividad y diversidad variaron entre sitios. Los humedales herbáceos más diversos están en la cuenca baja del Papaloapan, seguidos por Ciénaga del Fuerte, en la planicie de inundación del Río Tecolutla. La cuenca baja del Papaloapan tiene una planicie costera inundable extensa y de alta frecuencia de inundaciones, presentándose 3.5 veces por año (OrtizPérez et al., 1991) con grandes superficies de complejos de comunidades de humedales. En este sentido aparece la 
tendencia a una mayor diversidad de humedales herbáceos emergentes en zonas extensas, que forman planicies inundables con marcada fluctuación de los niveles de inundación en comparación con hondonadas (La Mancha) o planicies internas (Laguna Apompal) donde el hidroperíodo es más homogéneo a lo largo del año. El humedal de Río Blanco es el menos diverso del grupo de humedales emergentes del Papaloapan, y florísticamente difiere del resto por la presencia de individuos arbustivos como Dalbergia brownei, Malvaviscus arboreus, Pithecellobium calostachys y Acacia cornigera. La presencia de elementos leñosos en humedales herbáceos puede ser interpretada como individuos sobrevivientes de un área mayor de humedal con elementos arbóreos, o como organismos que germinaron in situ, y es frecuente encontrar asociaciones de leguminosas en zonas de humedales (Darch, 2000). Cabe mencionar que estos valores de diversidad son bastante menores que los reportados para los humedales herbáceos emergentes del Altiplano (Rzedowski, 1983).

Los hidroperíodos identificados para cada humedal mostraron que estas diferencias ambientales también contribuyen a mantener la variedad de estos tipos de comunidades en la planicie del Golfo de México, como ha sido demostrado por Yetter (2004) y Moreno-Casasola et al. (2009), quienes encuentran para el complejo de los humedales de La Mancha que el nivel de inundación es uno de los principales factores que diferencia a las comunidades. En el presente trabajo también se encuentran diferencias en los niveles y periodicidad de la inundación entre los distintos tipos de humedales herbáceos. Typha domingensis es una especie dominante en ambas agrupaciones y constituye una de las hidrófitas de más amplia distribución bajo diversas condiciones ambientales y con mayor tolerancia a una variedad de factores (Richardson et al., 2008). Además se le encuentra en lagos inundados permanentemente. Orozco-Segovia y Lot-Helgueras (1976) midieron la fluctuación de agua en los humedales del sureste de Veracruz, encontrando que las raíces de las plantas permanecían inundadas todo el año, ya que solo durante dos meses el agua bajaba a nivel del suelo.

Factores como la microtopografía y la humedad del suelo influyen en el establecimiento de las especies, siendo éstos determinantes para la formación de las comunidades de humedales (Rey Benayas et al., 1999; Lathrop et al., 2003; López Rosas et al., 2005; Flores-Verdugo et al., 2007; Hopfensperger y Engelhardt, 2008; Farruggia, 2008). De acuerdo a los resultados del contenido de humedad en el horizonte más superficial del suelo, Ciénaga del Fuerte retiene más agua, mientras que el humedal emergente de Río Limón la menor, en parte debido al manejo ganadero al que está sometido. La mayoría de los humedales herbáceos estudiados mantienen el suelo saturado en la temporada de estiaje, lo que se relaciona con las características físicas que presentan. El horizonte orgánico, de hasta $40 \mathrm{~cm}$ de espesor, tiene una densidad aparente muy baja (entre 0.1 y $0.3 \mathrm{~g} \mathrm{~cm}^{-3}$ ) y una alta porosidad (81 a 93\%) que favorece tanto el flujo como la retención de agua. Sin embargo en los horizontes inferiores (en ocasiones minerales), la densidad aparente aumenta a valores de $1.29 \mathrm{~g} \mathrm{~cm}^{-3}$, lo que hace que el flujo vertical del agua disminuya significativamente, y el horizonte orgánico se mantenga más tiempo saturado de agua (datos no publicados), que se ve reflejado en el contenido de humedad reportado (Pw) en el cuadro 1.

Se sabe que los humedales herbáceos pueden establecerse sobre suelos minerales (Shiflet, 1963) o de origen orgánico (Neubauer, 2008). Esta última condición se presenta para la mayor parte de los humedales en estudio, especialmente de la cuenca baja del Papaloapan, donde se describen los humedales con más especies y los más diversos, los cuales se desarrollan sobre Histosoles. Sin embargo no se tiene aún suficiente información para poder establecer una relación entre el tipo de suelo y el tipo de humedal.

No hay una relación clara entre los parámetros físicoquímicos del agua intersticial y las especies o agrupaciones florísticas. En general Río Blanco, Río Limón y Mandinga presentaron los valores más altos de conductividad y salinidad indicando una mayor influencia de la cuña salina y materiales en solución, debido probablemente a su asociación con ríos y escurrimientos de las porciones medias y altas de su respectiva cuenca, o escurrimientos intracuenca. $\mathrm{El} \mathrm{pH}$ no mostró grandes diferencias, con valores alrededor de 7 en todos los humedales. El nivel de inundación y el hidroperiodo fueron los parámetros ambientales que mayores diferencias presentaron a nivel de sitio.

Particularidades de algunas especies de los humedales herbáceos. Ciénaga del Fuerte presenta especies poco comunes en otros humedales herbáceos. Boehmeria cylindrica es una Urticaceae muy flexible a cambios en su ambiente (Woodward, 1993), ampliamente distribuida en bosques húmedos y humedales de Estados Unidos (Wilmot Dear y Friis, 1996) llegando a formar colchones flotantes. También forma parte de humedales en Sudamérica y tiene aplicaciones médicas (Al-Shamma et al., 1982). Vigna luteola, de origen africano, es una trepadora presente en humedales herbáceos, altamente palatable y productiva usada para forraje (Ludlow y Wilson, 1971; Sasser y Gosselink, 1984). Luziola peruviana pertenece a un pequeño género de gramíneas que habita ambientes acuáticos de poca profundidad, que se reproduce sexual y asexualmente, y sirve de alimento a fauna silvestre como los capibaras (Quintana et al., 1998), además se aprovecha como alimento para ganado (Pereira et al., 2003) $y$ en algunos sitios se ha convertido en una plaga que se ha buscado controlar mediante carpas (Sponchiado y Schwarzbold, 2009). Leersia es un género de gramínea asociado a zonas inundables, no tolera sequías prolongadas, y facilita el establecimiento de comunidades vegetales (De Steven y Sharitz, 2007).

Hymenachne amplexicaulis, un pasto de los humedales, 
aparece con valores altos en algunos humedales emergentes donde se introduce ganado y es empleado en áreas inundables o de baja disponibilidad de nitrógeno, especialmente para producción de leche (Enríquez-Quiroz et al., 2006), razón por la cual su propagación se ve favorecida localmente. Esta especie puede desplazar a otras especies nativas del humedal (Houston y Duivenvoorden, 2002) y ha sido catalogada como la principal amenaza a humedales en otros países fuera de su ámbito de distribución, como Australia (Dry Tropics Biodiversity Group Inc.). Zizaniopsis miliacea es otra gramínea de humedales, importante para la protección de fauna. Entre los pastos registrados, Cynodon dactylon y Echinochloa pyramidalis son introducidos (Valdes-Reyna y Dávila, 1995; López Rosas et al., 2006).

En el humedal de la Popotera se observa vegetación flotante dominada por Eichhornia crassipes, Pontederia sagittata, Hymenachne amplexicaulis y Thalia geniculata. Son grandes masas vegetales que se desplazan sobre el agua, formando densas alfombras de materia orgánica en la superficie del río, que con el tiempo se depositan en el fondo. Las masas vegetales se forman en las orillas de los canales, sobre materia orgánica muerta de plantas anuales. Con el aumento de inundación y corrientes en época de lluvias, se desprenden y se desplazan con las corrientes hacia desembocaduras de ríos de mayor orden. Cuando se aglutinan o retienen en zonas de poca profundidad, se convierten en superficies sin sustrato consolidado conocidas localmente como "tembladeras".

Actividades humanas que promueven cambio de uso de suelo en humedales herbáceos. Algunos autores reportan asociaciones entre especies de hidrófitas herbáceas emergentes y pastos forrajeros tolerantes a la inundación como el caso de la asociación de Cynodon dactylon-Sagittaria lancifoliaIpomoea carnea- Setaria geniculata, también la de Cyperus articulatus- Paspalum notatum- Hydrocotyle bonariensis con algunos cuadros con Cynodon dactylon, la asociación de Setaria geniculata y como especie acompañante Thalia geniculata y la de Echinochloa pyramidalis (Orozco-Segovia y Lot-Helgueras, 1976; Moreno-Casasola et al., 2009; Peralta-Peláez y Moreno-Casasola, 2009). Ello habla de la invasión en algunos casos, o de la introducción en otros, de gramíneas exóticas a los humedales para incrementar su productividad para el pastoreo de ganado en zonas inundables.

En la zona de centro - norte de Veracruz no se forman planicies de inundación tan extensas como en el sur de Veracruz o en la zona baja del Río Pánuco, y los humedales emergentes ocupan superficies menores y son mucho más pobres en especies, con excepción de Ciénaga del Fuerte. Están aislados unos de otros, rodeados por una matriz formada por otros tipos de humedales arbóreos, como selvas inundables y manglares, y por potreros o campos agrícolas (caña de azúcar y frutales principalmente). Muchos de ellos han sido convertidos en potreros cultivados que se inundan durante la época de lluvias o terrenos que se drenan para actividades productivas. Presentan especies que indican uso ganadero o de perturbación humana como lo son Mimosa pigra (Lot-Helgueras y Novelo, 1990) y la gramínea exótica E. pyramidalis, que ha sido introducida en los humedales para transformarlos en potreros para ganadería (López Rosas et al., 2006) con graves consecuencias a la hidrología y la composición de especies. Algunos humedales pueden ser invadidos por especies introducidas o por especies nativas que se propagan y colonizan el humedal rápidamente convirtiéndose en problemáticas, como Phragmites spp. (Lathrop et al., 2003), T. domingensis (Osland, 2009) y E. pyramidalis (Lopez-Rosas, 2007). Estas especies presentan una propagación vegetativa por rizomas lo que les permite expandirse y convertir un humedal diverso en uno monoespecífico. El humedal que permanece menos tiempo inundado es el que presenta E. pyramidalis (grupo c), es decir, un humedal herbáceo que es invadido por una especie usada para forraje de ganado, puede inducir que popales o tulares cambien su composición florística y funcionamiento y muestren aún mayor predisposición a cambios de uso de suelo (López Rosas, 2007). El VIR de esa especie (mostrado en Apéndice 1) es relativamente alto en algunas zonas, y alguna modificación en la comunidad o cambios en el régimen de inundación, podría permitir un rápido crecimiento y traer cambios en la composición de especies.

Variabilidad florística y el uso de los términos popal y tular. El presente trabajo, así como la literatura reportada, muestran una gran variabilidad en la composición florística de los humedales de distintas zonas. Las explicaciones de ello pueden ser de varios tipos. Por un lado, están las diferencias regionales sobre todo en las geoformas e hidrología de las zonas, lo cual determina las especies que pueden sobrevivir a hidroperiodos particulares. Por otro están las colindancias con otros humedales o comunidades que pueden aportar especies particulares a cada humedal incluyendo especies invasoras potencialmente. Muchas de estas colindancias están en función del manejo y uso del humedal. La mayoría de los humedales herbáceos emergentes han estado sujetos a algún tipo de uso, principalmente ganadería, durante la cual hay modificaciones al drenaje, quemas y distintas cargas ganaderas. Todos estos factores funcionan como filtros que van determinando la entrada y erradicación de especies localmente. Además existen mecanismos de regeneración o factores ambientales que generan cambios en la estructura y diversidad de la vegetación de los humedales. Entre ellos está la variación de la salinidad y los nutrientes a lo largo del año, sea por la entrada de agua dulce de las lluvias o por escurrimientos, los cuales son promotores de cambios en la estructura vegetal (Bedford et al., 1999; Dunton et al., 2001). Typha domingensis tolera un nivel mayor de salinidad (Glenn et al., 1995), más que otras especies de humedales herbáceos emergentes. También se debe tener en cuen- 
ta al banco de semillas del suelo y el banco de rizomas y estolones que favorece la regeneración de algunas especies sobre otras cuando la vegetación existente es removida (López Rosas et al. 2006; Osland, 2009) o por algún disturbio natural (Davis et al., 2004). La capacidad de crecimiento vegetativo de muchas de estas especies es un factor que probablemente favorece su dominancia.

Los humedales herbáceos emergentes forman un gradiente desde los dominados por una especie hasta los ricos en monocotiledóneas y dicotiledóneas. Se distinguen principalmente por la fisonomía foliar de la especie dominante, presentándose en un extremo los tulares, carrizales y los humedales invadidos por gramíneas y en el otro los popales. Sin embargo de manera natural forman un gradiente complejo en el paisaje caracterizado por asociaciones de especies con distinto grado de dominancia como se discutió anteriormente. En este sentido se pueden encontrar verdaderos tulares (casi monoespecíficos), popales, carrizales y comunidades de ciperáceaes, aunque predomina la mezcla de ellos formando un complejo de humedales de composición específica variable. En este sentido se sugiere continuar utilizando la denominación del binomio popal -tular (esta última incluyendo las comunidades de ciperáceas y los carrizales) para referirse a este complejo variable.

La composición florística de popales-tulares de la planicie costera central de Veracruz es variable y diversa. La mayor diversidad se encuentra en humedales herbáceos de la cuenca baja del Papaloapan y del Río Tecolutla, sobre las planicies de inundación, por lo que se establece un gradiente latitudinal en la diversidad. Los sistemas de humedales herbáceos del centro y norte del Estado se encuentran fraccionados y aislados por efecto de actividades agrícolas y ganaderas, principalmente, lo cual también reduce su diversidad.

De acuerdo al análisis de clasificación se tipificaron los humedales herbáceos en nueve grupos que varían en su riqueza y composición florística, aunque comparten especies, diferenciándose también por las condiciones de inundación y el contenido de humedad del suelo más superficial. Cinco de estos grupos se presentan en un solo sitio y cuatro en varios sitios. Ello probablemente es un reflejo de la importancia de condiciones locales como la vecindad de otros humedales, la historia de colonización y manejo, entre otras. La existencia de pastos forrajeros y la predisposición de humedales herbáceos para ser colonizados por estas especies, hace que estos ecosistemas inundables sean vulnerables a un proceso de "potrerización", mediante prácticas como la desecación de humedales y la introducción y siembra de especies forrajeras.

Los parámetros medidos en el agua intersticial presentaron diferencias en $\mathrm{pH}$, salinidad, conductividad, máximo nivel de inundación, tipo de suelos y humedad del mismo. Los hidroperiodos mostraron tres grupos, relacionados con las agrupaciones florísticas definidas previamente. El pri- mero corresponde a las agrupaciones que pasan la mayor parte del año inundadas, el segundo a aquellas en las que el agua se mantiene por encima del suelo durante varios meses del año, aunque durante la temporada de secas se reduce el nivel del agua y el tercero corresponde a los grupos en los que una buena parte del año el agua se encuentra por debajo del nivel del suelo. La ordenación puede interpretarse como gradientes de diversidad y de tipo de suelo, predominando los histosoles en los humedales del Papaloapan y los gleysoles en la parte centro y norte del Estado.

El conjunto de humedales herbáceos emergentes y las variantes potrerizadas se organizan en un gradiente de dominancia/riqueza que forma un complejo paisajístico. En este sentido se sugiere utilizar la denominación popal-tular (incluyendo las comunidades de ciperáceas y los carrizales) para referirse a este complejo variable. Son comunidades que brindan servicios ambientales importantes a la sociedad, por lo que se debe buscar su conservación restauración.

\section{Agradecimientos}

El presente trabajo fue financiado por el proyecto Humedales del Papaloapan CONACYT-CONAGUA (48247), proyecto ITTO PD 349/05 Rev.2 (F), y el Instituto de Ecología A.C. (902-17). Se agradece a Ariadna Martínez y Lourdes Cruz por el apoyo en el procesamiento de muestras, a Rosario Landgrave por su ayuda en el uso e interpretación de cartografía, Roberto Monroy por las figuras y Graciela Sánchez por la revisión, a Light Hawk por su gran apoyo. Deseamos agradecer a un revisor anónimo sus comentarios los cuales ayudaron a enriquecer la discusión del artículo.

\section{Literatura citada}

Al-Shamma A., Drake S.D., Guagliardi L.E., Mitscher L.A. y Swayze J.K. 1982. Antimicrobial alkaloids from Boehmeria cylindrica. Phytochemistry 21:485-487.

Bedford B.L., Walbridge M.R. y Aldous A. 1999. Patterns in nutrient availability and plant diversity of temperate North American wetlands. Ecology 80:2151-2169

Cházaro M. 1986. La vegetación. Serie Medio Ambiente en Coatzacoalcos 6. Centro de Ecodesarrollo, México, D.F. México.

Collins P.E.F., Turner S.D. y Cundy A.B. 2001. High-resolution reconstruction of recent vegetation dynamics in a mediterranean microtidal wetland: implications for site sensitivity and palaeoenvironmental research. Journal of Coastal Research 17:684-693.

Costanza R., d'Arge R., de Groot R., Farber S., Grasso M., Hannon B., Limburg K., Naeem S., O’Neill R.V., Paruelo J., Raskin R.G., Sutton P. y van den Belt M. 1998. The value of the world's ecosystem services and natural capital. Ecological Economics 25:3-15.

Darch J.P. 2000. Vegetation associations at Pulltrouser swamp. En: Turner II B.L. y Harrison P.D. Eds. Pulltrouser swamp: Ancient Maya habitat, agriculture, and settlement in northern Belize, pp.21-29. University of Texas Press. Austin. 
Davis III S.E., Cable J.E., Childers D.L., Coronado-Molina C., Day Jr. J.W., Hittle C.D., Madden C. J., Reyes E., Rudnick D. y Sklar F. 2004. Importance of storm events in controlling ecosystem structure and function in a Florida gulf coast estuary. Journal of Coastal Research 20:1198-1208.

De Steven D. y Sharitz R. 2007. Transplanting native dominant plants to facilitate community development in restored coastal plain wetlands. Wetlands 27:972-978.

Diego-Pérez N., Fonseca R.M., Pérez-Lozada L. y Lorea-Hernández F. 1993. Vegetación de las lagunas costeras y zonas inundables del Estado de Guerrero, México. Brenesia 39-40:7-28.

Diego-Pérez N. y Lozada-Pérez L. 1994. Laguna de Tres Palos. En: Diego-Pérez N. y Fonseca R.M. Eds. Estudios florísticos en Guerrero, No. 3. Las Prensas de Ciencias, Facultad de Ciencias, Universidad Nacional Autónoma de México. México, D.F., México.

Doren, R.F., Armentano T.V., Whiteaker L.D. y Jones R.D 1997. Marsh vegetation patterns and soil phosphorus gradients in the Everglades ecosystem. Aquatic Botany 56:145-163.

Dry Tropics Biodiversity Group, Inc.

<www.drytropics.org.au/weeds_wetlandweed_strat.htm\#top> (consulta el 12 de enero de 2010)

Dunton K.H., Hardegree B. y Whitledge T.E. 2001. Response of estuarine marsh vegetation to interannual variations in precipitation. Estuaries 24:851-861.

Ellison A. 2004. Wetlands of Central America. Wetlands Ecology and Management 12:3-55.

Enríquez-Quiroz J.F., Quero-Carrillo A.R., Hernández-Garay A. y García-Moya E. 2006. Azuche, Hymenachne amplexicaulis (Rudge) Nees, forage genetic resources for floodplains in tropical Mexico. Genetic Resources and Crop Evolution 53:14051412.

Ewe S.M.L., Gaiser E.E., Childers D.L., Iwaniec D., Rivera-Monroy V.H. y Twilley R.R. 2006. Spatial and temporal patterns of aboveground net primary productivity (ANPP) along two freshwater-estuarine transects in the Florida coastal Everglades. Hydrobiologia 569:459-474.

Farruggia F.T., Stevens M.H.H. y Vincent M.A. 2008. A floristic description of a Neotropical coastal savanna in Belize. $\mathrm{Ca}$ ribbean Journal of Science 44:53-69.

Finlayson C.M., Davidson N.C., Spiers A.G. y Stevenson N.J. 1999. Global wetland inventory - current status and future priorities. Marine and Freshwater Research 50:717-727.

Flores-Verdugo F., Moreno-Casasola P., Agraz-Hernández C., López-Rosas H., Benítez-Pardo D. y Travieso-Bello A. 2007. La topografía y el hidroperíodo: dos factores que condicionan la restauración de los humedales costeros. Boletín de la Sociedad Botánica de México 80S:33-47.

Fonseca R.M. y Lozada L. 1993. Laguna de Coyuca. En: DiegoPérez N. y Fonseca R.M. Eds. Estudios florísticos en Guerrero, No. 1. Las Prensas de Ciencias, Facultad de Ciencias, Universidad Nacional Autónoma de México. México, D.F. México.

Fortney R.H., Benedict M., Gottgens J.F., Walters T.L., Leady B.S. y Rentch J. 2004. Aquatic plant community composition and distribution along an inundation gradient at two ecologicallydistinct sites in the Pantanal region of Brazil. Wetlands Ecology and Management 12:575-585.

Glenn E., Thompson T.L., Frye R., Riley J. y Baumgartner D. 1995. Effects of salinity on growth and evapotranspiration of Typha domingensis Pers. Aquatic Botany 52:75-91.
Greig-Smith P. 1983. Quantitative plant ecology. Third edition. Volume 9 in Studies in Ecology. University of California Press, Berkeley.

Gutiérrez B.C. y. Zolá M.G. 1987. Hidrófitas de Nevería, Veracruz, México. Biotica 12:21-34.

Hopfensperger K.N. y Engelhardt K.A.M. 2008. Annual species abundance in a tidal freshwater marsh: germination and survival across an elevational gradient. Wetlands 28:521-526.

Houston W.A. y Duivenvoorden L.J. 2002. Replacement of littoral native vegetation with the ponded pasture grass Hymenachne amplexicaulis: effects on plant, macroinvertebrate and fish biodiversity of backwaters in the Fitzroy River, Central Queensland, Australia. Marine and Freshwater Research 53:12351244.

INEGI [Instituto Nacional de Estadística, Geografía e Informática]. 2005. Conjunto de datos vectoriales de la carta de uso del suelo y vegetación: escala 1:250 000. Serie III (continuo nacional). Instituto Nacional de Estadística, Geografía e Informática. México.

Infante Mata D., Peralta-Peláez L.A. y Arrocha A.A. 2009. Obtención de datos de salinidad, conductividad y pH del agua. En: Moreno-Casasola P. y Warner B. Eds. Breviario para describir, observar y manejar humedales, pp. 31-42. Serie Costa Sustentable 1. RAMSAR, Instituto de Ecología A.C., CONANP, US Fish and Wildlife Service, US State Department. Xalapa.

IUSS [International Union of Soil Sciences Working GroupWRB]. 2006. World reference base for soil resources. World Soil Resources Reports No. 103. FAO, Roma. Italia.

Kandus P. y Adamoli J.M. 1993. Freshwater marsh vegetation response to flooding patterns in the lower delta of the Parana River. Wetlands Ecology and Management 2:213-222.

Lathrop R., Windham L. y Montesano P. 2003. Does Phragmites expansion alter the structure and function of marsh landscapes? Patterns and processes revisited. Estuaries 26:423-435.

López-Rosas H. 2007. Respuesta de un humedal transformado por la invasión de la gramínea exótica Echinochloa pyramidalis Hitchc. \& A. Chase a los disturbios inducidos (cambios en el hidroperíodo, apertura de espacios y modificación de la intensidad lumínica). Tesis doctoral. Instituto de Ecología A.C., Xalapa, México. 134 pp.

López-Rosas H., Moreno-Casasola P. y Mendelssohn I.A. 2005. Effects of an African grass invasion on vegetation, soil and interstitial water characteristics in a tropical freshwater marsh in La Mancha, Veracruz (Mexico). Journal of Plant Interaction 1:187-195.

López-Rosas H., Moreno-Casasola P. y Mendelssohn I.A. 2006. Effects of experimental disturbances on a tropical freshwater marsh invaded by the African grass Echinochloa pyramidalis. Wetlands 26:593-604.

López-Rosas H., López-Barrera F., Moreno-Casasola P., AguirreLeón G., Cázares-Hernández E. y Sánchez-Higueredo L. 2010. Indicators of recovery in a tropical freshwater marsh invaded by an African grass. Ecological Restoration 28:324-332.

Lorenzen B., Brix H., Mendelssohn I.A., McKee K.L. y Li-Miao S.L. 2001. Growth, biomass allocation and nutrient use efficiency in Cladium jamaicense and Typha domingensis as affected by phosphorus and oxygen availability. Aquatic Botany 70:117-133.

Lot A. y Novelo A. 1988b. Vegetación y flora acuática del Lago de Pátzcuaro, Michoacán. Southwestern Naturalist 33:167-175. 
Lot-Helgueras A. 1991. Flora y vegetación de las zonas acuáticas de Veracruz. Tesis doctoral. Facultad de Ciencias, Universidad Nacional Autónoma de México, México D.F. 226 pp.

Lot-Helgueras A. 2004. Flora and vegetation of freshwater wetlands in the coastal zone of the Gulf of Mexico. En: Caso M., Pisanty I. y Ezcurra E. Eds. Environmental Analysis of the Gulf of Mexico, pp. 314-339. SEMARNAT - Instituto Nacional de Ecología - Instituto de Ecología, A.C. - Harte Research Institute for Gulf of Mexico Studies - Texas A\&M University, México D.F.

Lot-Helgueras A. y Novelo A. 1988a. El pantano de Tabasco y Campeche: la reserva más importante de plantas acuáticas de Mesoamérica. En: Anónimo. Ecología y conservación del delta de los ríos Usumacinta y Grijalva, Memorias. pp 537-547. INIREB-División Regional Tabasco, Gobierno del Estado de Tabasco. Villahermosa.

Lot-Helgueras A. y Novelo A. 1990. Forested Wetlands of Mexico. En: Lugo A.E., Brinson M.M.y Brown S. Eds. Forested wetlands of the World (Ecosystems of the World), pp. 287-298. Elsevier Publ. Co. Amsterdam.

Ludlow M.M. y Wilson G.L. 1971. Photosynthesis of tropical pasture plants. 2. Temperature and illuminance history. Australian Journal of Biological Sciences 24:1065-1075.

Martínez M. y Novelo A. 1993. La vegetación acuática del estado de Tamaulipas, México. Anales del Instituto de Biología UNAM. Serie Botánica 64:59-86.

McCune B. y Mefford M. 2006. PC-ORD. Multivariate Analysis of Ecological Data. Version 5. MjM Software, Gleneden Beach, Oregon.

McCune B., Grace J.B. y Urban D.L. 2002. Analysis of ecological communities. MJM Software Design, Gleneden Beach, Oregon.

McKee K.L, Mendelssohn I.A, y Hester M.W. 1988. A reexamination of pore water sulfide concentrations and redox potentials near the aerial roots of Rhizophora mangle and Avicennia germinans. American Journal of Botany 75:1352-1359.

Miranda F. y Hernández-X. E. 1963. Los tipos de vegetación de México y su clasificación. Boletín de la Sociedad Botánica México 28:29-178.

Mitsch W.J. y Gosselink J.G. 2000. Wetlands. J. Wiley and Sons Inc. Nueva York.

Moreno-Casasola P., López-Rosas H., Infante-Mata D., Peralta L.A., Travieso-Bello A.C. y Warner B.G. 2009. Environmental and anthropogenic factors associated with coastal wetland differentiation in La Mancha, Veracruz, Mexico. Plant Ecology 200:37-52.

Neubauer S.C. 2008. Contributions of mineral and organic components to tidal freshwater marsh accretion. Estuarine, Coastal and Shelf Science 78:78-88.

Novelo R. y Ramos L. 2005. Vegetación acuática. En: Bueno J., Álvarez F., Santiago S. Eds. Biodiversidad del estado de Tabasco, pp. 11-144. Instituto de Biología, UNAM-CONABIO. México D.F.

Ocaña D. y Lot A.H. 1996. Estudio de la vegetación acuática vascular del sistema fluvio-lagunar-deltaico del Río Palizada en Campeche, México. Anales del Instituto de Biología, UNAM. Serie Botánica 67: 303-327.

Olmsted I. 1993. Wetlands of Mexico. En: Whigham D.F., Dykyjová D. y Hejnÿ S. Eds. Wetlands of the world I: Inventory, ecology and management, pp. 637-678. Handbook of Vegetation
Science. Kluwer Academic Publishers, Dordrcht.

Orozco-Segovia A. 1974. Estudio de la vegetación y flor de zonas inundables del sur de Veracruz. Tesis Profesional. Facultad de Ciencias, Universidad Nacional Autónoma de México. México D.F. 117 pp.

Orozco- Segovia A. y Lot-Helgueras A. 1976. La vegetación de las zonas inundables del sureste de Veracruz. Biotica 1:1-44.

Ortíz-Pérez M. Oropeza-Orozco O., Palacio-Aponte A., D’LunaFuentes A. 1991. Zonas susceptibles a desastres por fenómenos naturales: inundaciones. En: Atlas Nacional de México. Instituto de Geografía - Universidad Nacional Autónoma de México, México D.F.

Osland M. J. 2009. Managing invasive plants during wetland restoration: the role of disturbance, plant strategies, and environmental filters. Tesis Doctoral. Environmental Sciences. Duke University. $181 \mathrm{pp}$.

Padial A.A., Carvalho P., Thomaz S.M., Boschilia S.M., Rodrigues R.B. y Kobayashi J.T. 2009. The role of an extreme flood disturbance on macrophyte assemblages in a Neotropical floodplain. Aquatic Sciences 71:389-398

Peralta-Peláez L.A. y Moreno-Casasola P. 2009. Composición florística y diversidad de la vegetación de humedales en los lagos interdunarios de Veracruz. Boletín de la Sociedad Botánica de México 85:89-101.

Peralta-Peláez L.A., Infante-Mata D. y Moreno-Casasola P. 2009. Construcción e instalación de piezómetros. En: Moreno-Casasola P. y Warner B. Eds. Breviario para describir, observar y manejar humedales, pp. 17-30. Serie Costa Sustentable 1. RAMSAR, Instituto de Ecología A.C., CONANP, US Fish and Wildlife Service, US State Department. Xalapa.

Pereira J.A., Quintana R.D. y Monge S. 2003. Diets of plains vizcacha, greater rhea and cattle in Argentina. Journal of Range Management 56:13-20.

Pérez L.A., Sousa M., Hanan A.M., Chiang F. y Tenorio P. 2005. La vegetación terrestre. En: Bueno J., Álvarez F. y Santiago S. Eds. Biodiversidad del estado de Tabasco, pp. 65-110. CONABIO-Instituto de Biología, UNAM. México, D.F.

Quintana R.D., Monge S. y Malvárez A.I. 1998. Feeding patterns of capybara Hydrochaeris hydrochaeris (Rodentia, Hydrochaeridae) and cattle in the non-insular area of the Lower Delta of the Paraná River, Argentina. Mammalia 62:37-52.

Rey-Benayas J.M., Colomer M.G.S. y Levassor C. 1999. Effects of area, environmental status and environmental variation on species richness per unit area in Mediterranean wetlands. Journal of Vegetation Science 10:275-280.

Richardson C.J., King R.S., Vymazal J., Romanowicz E.A. y Pahl J.W. 2008. Macrophyte community responses in the Everglades with an emphasis on cattail (Typha domingensis) and sawgrass (Cladium jamaicense) interactions along a gradient of longterm nutrient additions, altered hydroperiod, and fire. En: Richardson C.J. Ed. The Everglades experiments studies. Lessons for ecosystem restoration, pp. 215-260. Ecological Studies Vol 201. Springer. Nueva York.

Rojas-Moreno J. y Novelo-Retana A. 1995. Flora y vegetación acuáticas del Lago de Cuitzeo, Michoacán, México. Acta Botánica Mexicana 31:1-17.

Rzedowski, J. 1983. Vegetación de México. Limusa, México D.F.

Sasser C.E. y Gosselink J.G. 1984. Vegetation and primary production in a floating freshwater marsh in Louisiana. Aquatic Botany 20:245-255. 
Semlitsch R.D. y Bodie J.R. 1998. Are small, isolated wetlands expendable? Conservation Biology 12:1129-1133.

Shannon C.E. y Weaver S. 1949. The mathematical theory of communication. The University of Illinois Press, Urbana.

Shiflet T.N. 1963. Major ecological factors controlling plant communities in Louisiana marshes. Journal of Range Management 16:231-235.

Soil Science Laboratory Manual. EVSC 427. Department of Environmental Sciences. The University of Virginia. Charlottesville. $</$ www.evsc.virginia.edu/ alm7d/soils/man.pdf> (consultado 7 de febrero de 2010)

Soto M. 1986. Localidades y climas del estado de Veracruz. INIREB. Xalapa.

Sponchiado M. y Schwarzbold A. 2009. Control of the aquatic macrophyte Luziola peruviana Juss. ex Gmel by grass carp (Ctenopharyngodon idella Valenciennes, 1844) grazing. Acta Limnologica Brasileira 21:193-197.

StatSoft, Inc. 1998. STATISTICA for Windows (Computer program manual). Tulsa, OK: StatSoft, Inc. Tulsa, Oklahoma.

Travieso-Bello A.C., Moreno-Casasola P. y Campos A. 2005. Efecto de diferentes manejos pecuarios sobre el suelo y la vegetación en humedales transformados a pastizales. Interciencia 30:12-18.

Valdes Reyna J. y Dávila P.D. 1995. Clasificación de los géneros de gramíneas (Poaceae) Mexicanas. Acta Botánica Mexicana 33:37-50.

Recibido: 8 de diciembre de 2009

Aceptado: 21 de octubre del 2010
Vázquez-Yánez C. 1971. La vegetación de la Laguna de Mandinga, Veracruz. Anales del Instituto de Biología UNAM. Serie Botánica 42:49-94.

Visser J.M., Sasser C.E., Chabreck R.H. y Linscombe R.G. 1998. Marsh vegetation types of the Mississippi River deltaic plain. Estuaries 21:818-828.

Westhoff V. y van der Maarel E. 1978. The Braun-Blanquet approach. En: Whittaker R. Ed. Classification of plant communities, pp. 287-399. Dr. W. Junk Publishers. Dordrecht.

Wheeler B.D., Money R.P. y Shaw S.C. 2002. Freshwater wetlands. En: Perrow M.R. y Davy A.J. Eds. Handbook of Ecological restoration, pp. 325-354. Vol. 2. Restoration in practice. Cambridge University Press. Cambridge.

Wilmot-Dear C.M. y Friis I. 1996. The new world species of Boehmeria and Poulzolzia (Urticaceae, tribu Boehmeriaceae). A taxonomic revision. Opera Botanica 129:1-103.

Woo I. y Zedler J.B. 2002. Can nutrients alone shift a sedge meadow towards dominance by the invasive Typha $\times$ glauca? Wetlands 22:509-521.

Woodward F.I. 1993. Plant responses to past concentrations of $\mathrm{CO}_{2}$. Plant Ecology 104-105:145-155.

Yetter J. 2004. Hydrology and geochemistry of freshwater wetlands on the Gulf coast of Veracruz, Mexico. Tesis Maestría. Earth Sciences. University of Waterloo. Canadá. 168 pp.

Zar J.H. 1984. Biostatistical analysis. Prentice-Hall. Englewood Cliffs. 
Apéndice 1. Lista de especies y su distribución en el complejo popal-tular muestreado. El valor numérico por sitio indica el Valor de Importancia Relativa en cada localidad y la última columna indica el Valor de Importancia Relativa global de cada especie. $\mathrm{FB}=$ Forma biológica, $\mathrm{A}=$ Árbol, $\mathrm{Ar}=$ Arbusto, $\mathrm{H}=$ Hierba, $\mathrm{B}=$ Bejuco. Forma de crecimiento (F Crec): EHF= Enraizada hojas flotantes, $\mathrm{EE}=$ Enraizada emergente, LF= Libre flotadoras. Ciénaga del Fuerte (CF), Estero Dulce (ED), Laguna Grande-Laguna Chica (LG-Ch), La Mancha (LM), Laguna Apompal (Apo), Laguna Mandinga (Man), Río Blanco popal (RBp), Río Blanco tular (RBt), Río Limón (RL), Laguna Conejo (Con), Sombrerete (Som), La Popotera (Pop). * denota las especies introducidas.

\begin{tabular}{|c|c|c|c|c|c|c|c|c|c|c|c|c|c|c|c|}
\hline & FB & F Crec & $\mathrm{CF}$ & ED & $\begin{array}{l}\text { LG- } \\
\text { Ch }\end{array}$ & LM & Apo & Man & RBp & RBt & $\mathbf{R L}$ & Con & Som & & $\begin{array}{l}\text { VIR } \\
\text { global }\end{array}$ \\
\hline \multicolumn{16}{|l|}{ PTERIDOPHYTAS } \\
\hline \multicolumn{16}{|l|}{ Marsileaceae } \\
\hline Marsilea polycarpa Hook. \& Grev. & $\mathrm{H}$ & EHF & & & & & & & & & & 3.3 & & & 1.08 \\
\hline \multicolumn{16}{|l|}{ Pteridaceae } \\
\hline Adiantum sp. & $\mathrm{H}$ & $\mathrm{EE}$ & 4.6 & & & & 10.8 & & & & & & & & 1.81 \\
\hline \multicolumn{16}{|l|}{ Salviniaceae } \\
\hline Salvinia minima Baker & $\mathrm{H}$ & LF & & & & 2.7 & & & & & 1.7 & & & & 1.64 \\
\hline \multicolumn{16}{|l|}{ MONOCOTILEDÓNEAS } \\
\hline \multicolumn{16}{|l|}{ Alismataceae } \\
\hline Sagittaria lancifolia L. & $\mathrm{H}$ & $\mathrm{EE}$ & & & 6.7 & 3.7 & & 28.2 & & 8.8 & 2.4 & 3.3 & & & 4.98 \\
\hline $\begin{array}{l}\text { Sagittaria lancifolia L. subsp. media } \\
\text { (Micheli) Bogin }\end{array}$ & $\mathrm{H}$ & $\mathrm{EE}$ & & & & 12 & & & & & & & & & 5.12 \\
\hline $\begin{array}{l}\text { Sagittaria latifolia Willd. var. latifolia } \\
\text { Amaryllidaceae }\end{array}$ & $\mathrm{H}$ & $\mathrm{EE}$ & & & & & & & & & & & 6.1 & 5.8 & 2.15 \\
\hline $\begin{array}{l}\text { Hymenocallis littoralis (Jacq.) Salisb. } \\
\text { Arecaceae }\end{array}$ & $\mathrm{H}$ & $\mathrm{EE}$ & & & & & & & & & & 1.8 & & & 0.89 \\
\hline $\begin{array}{l}\text { Attalea butyracea (Mutis ex L. f.) } \\
\text { Wess. Boer }\end{array}$ & A & $\mathrm{EE}$ & & & & & & & & & & & & 5.3 & 3.19 \\
\hline $\begin{array}{l}\text { Sabal mexicana Mart. } \\
\text { Commelinaceae }\end{array}$ & Ar & $\mathrm{EE}$ & & & & & & & 2.9 & & & & & & 0.86 \\
\hline $\begin{array}{l}\text { Commelina diffusa Burm. f. } \\
\text { Cyperaceae }\end{array}$ & $\mathrm{H}$ & $\mathrm{EE}$ & & & & & & & & & 1.2 & & & 4.5 & 1.3 \\
\hline Cyperus articulatus L. & $\mathrm{H}$ & $\mathrm{EE}$ & & & & & 3.9 & 26.3 & & 6.4 & 4.7 & 11 & & & 4.49 \\
\hline Cyperus giganteus Vahl & $\mathrm{H}$ & $\mathrm{EE}$ & & 23.2 & 33.5 & & & & & & & & 12.2 & 14.8 & 7.34 \\
\hline Cyperus hermaphroditus (Jacq.) Standl. & $\mathrm{H}$ & $\mathrm{EE}$ & & & & & & & & & 0.9 & & & & 0.69 \\
\hline Eleocharis cellulosa Torr. & $\mathrm{H}$ & $\mathrm{EE}$ & & & & & & & 2.9 & 17.5 & 3.3 & 3.6 & & & 3.67 \\
\hline Eleocharis elegans (Kunth) Roem. \& Schult. & $\mathrm{H}$ & $\mathrm{EE}$ & & & & & & & & & & 1.8 & & & 0.89 \\
\hline Fimbristylis spadicea (L.) Vahl & $\mathrm{H}$ & $\mathrm{EE}$ & & & & & & & & 11.1 & & & & & 1.44 \\
\hline $\begin{array}{l}\text { Scleria sp. } \\
\text { Dioscoreaceae }\end{array}$ & $\mathrm{H}$ & $\mathrm{EE}$ & & & & 3.8 & & & & & & & & 7.4 & 3.19 \\
\hline $\begin{array}{l}\text { Dioscorea convolvulacea Schltdl. \& Cham. } \\
\text { Limnocharitaceae }\end{array}$ & B & $\mathrm{EE}$ & 3.1 & & & & & & & & & & & & 0.89 \\
\hline $\begin{array}{l}\text { Limnocharis flava (L.) Buchenau } \\
\text { Marantaceae }\end{array}$ & $\mathrm{H}$ & $\mathrm{EE}$ & & & & & & & & & & & 14.6 & & 1.67 \\
\hline Maranta arundinacea L. & $\mathrm{H}$ & $\mathrm{EE}$ & & & & & 3.9 & & & & & & & & 0.68 \\
\hline $\begin{array}{l}\text { Thalia geniculata L. } \\
\text { Orchidaceae }\end{array}$ & $\mathrm{H}$ & $\mathrm{EE}$ & 5 & 42.7 & & 1.8 & & & 4.1 & & & & 12.2 & 6.7 & 7.97 \\
\hline $\begin{array}{l}\text { Bletia purpurea (Lam.) D.C. } \\
\text { Poaceae }\end{array}$ & $\mathrm{H}$ & $\mathrm{EE}$ & & & & & 16.5 & & & & & & & & 1.45 \\
\hline Cynodon dactylon (L.) Pers. * & $\mathrm{H}$ & $\mathrm{EE}$ & & & & & & & & & 8.6 & & & & 2.02 \\
\hline Echinochloa crus-pavonis (Kunth) Schult. & $\mathrm{H}$ & $\mathrm{EE}$ & & & & & & & & & 2.3 & & & & 0.9 \\
\hline
\end{tabular}


Apéndice 1. Continuación

\begin{tabular}{|c|c|c|c|c|c|c|c|c|c|c|c|c|c|c|c|}
\hline & FB & F Crec & $\mathrm{CF}$ & ED & $\begin{array}{l}\text { LG- } \\
\text { Ch }\end{array}$ & LM & Apo & Man & RBp & RBt & RL & Con & Som & Pop & $\begin{array}{l}\text { VIR } \\
\text { global }\end{array}$ \\
\hline $\begin{array}{l}\text { Echinochloa pyramidalis (Lam.) } \\
\text { Hitchc. \& Chase* }\end{array}$ & $\mathrm{H}$ & $\mathrm{EE}$ & & 21.3 & & 19.9 & & 6 & & & & & & & 10.33 \\
\hline Hymenachne amplexicaulis (Rudge) Nees & $\mathrm{H}$ & $\mathrm{EE}$ & & & & & & & 4.1 & & & & 6 & 13.3 & 3.54 \\
\hline Leersia ligularis Trin. var. ligularis & $\mathrm{H}$ & $\mathrm{EE}$ & & & & & & & 6.3 & 11.1 & 11 & 17 & & & 6.33 \\
\hline Leersia sp. & & & & 12.8 & 15.2 & & 22.7 & & & & & & & & 3.12 \\
\hline Luziola peruviana Juss. ex J.F. Gmel. & $\mathrm{H}$ & $\mathrm{EE}$ & 9.6 & & 3.1 & & & & & & & & & & 2.39 \\
\hline Panicum sp. 23612 & $\mathrm{H}$ & $\mathrm{EE}$ & & & & & & & & & & & & 2.9 & 1.04 \\
\hline Paspalum sp. 23495 & $\mathrm{H}$ & $\mathrm{EE}$ & & & & & & & & 8.2 & & & & & 1.3 \\
\hline $\begin{array}{l}\text { Setaria paniculifera (Steud.) } \\
\text { E. Fourn. ex Hemsl. }\end{array}$ & $\mathrm{H}$ & $\mathrm{EE}$ & 8.7 & & & & & & & & & & & & 1.98 \\
\hline Spartina spartinae (Trin.) Merr. ex Hitchc. & $\mathrm{H}$ & $\mathrm{EE}$ & & & & 1.1 & & & 7 & & & & & & 1.51 \\
\hline Zizaniopsis miliacea (Michx.) Döll \& Asch. & $\mathrm{H}$ & $\mathrm{EE}$ & & & & 2.8 & & & & & 14.1 & 5.9 & & & 4.11 \\
\hline \multicolumn{16}{|l|}{ Pontederiaceae } \\
\hline Eichhornia crassipes (Mart.) Solms & $\mathrm{H}$ & LF & & & & & & & & & & 2.1 & & & 0.95 \\
\hline Pontederia sagittata C. Presl & $\mathrm{H}$ & $\mathrm{EE}$ & & & & & 16.1 & 8.2 & & 3.6 & 0.9 & 8.7 & 24.3 & & 5.83 \\
\hline \multicolumn{16}{|l|}{ Typhaceae } \\
\hline Typha domingensis Pers. & $\mathrm{H}$ & $\mathrm{EE}$ & & & 35.9 & 20.4 & 8.8 & & & 6.4 & 6.6 & & 8.8 & 2.3 & 12.31 \\
\hline \multicolumn{16}{|l|}{ DICOTILEDÓNEAS } \\
\hline $\begin{array}{l}\text { Ruellia foetida Willd. } \\
\text { Amaranthaceae }\end{array}$ & $\mathrm{H}$ & $\mathrm{EE}$ & & & & 1.1 & & & & & & & & & 0.89 \\
\hline $\begin{array}{l}\text { Alternanthera obovata (M. Martens } \\
\text { \& Galeotti) Millsp. }\end{array}$ & $\mathrm{H}$ & $\mathrm{EE}$ & & & & & & & & & & 1.8 & & & 0.89 \\
\hline Amaranthus dubius Mart. ex Thell. & $\mathrm{H}$ & $\mathrm{EE}$ & & & & & & & & & 2.6 & & & & 0.88 \\
\hline Amaranthus greggii S. Watson & $\mathrm{H}$ & $\mathrm{EE}$ & & & & & & & & 2.9 & & & & & 0.79 \\
\hline \multicolumn{16}{|l|}{ Asclepiadaceae } \\
\hline \multicolumn{15}{|l|}{ Asteraceae } & 1.76 \\
\hline $\begin{array}{l}\text { Archibaccharis serratifolia (Kunth) } \\
\text { S.F. Blake }\end{array}$ & $\mathrm{B}$ & $\mathrm{EE}$ & & & & & & & & & 1.7 & & & & 0.79 \\
\hline Eclipta prostrata (L.) L. & $\mathrm{H}$ & $\mathrm{EE}$ & & & & & & & & 2.9 & 0.9 & & & & 0.88 \\
\hline Sphagneticola trilobata (L.) Pruski & $\mathrm{H}$ & $\mathrm{EE}$ & & & & & & & & & & 1.2 & & & 0.78 \\
\hline \multicolumn{16}{|l|}{ Caesalpiniaceae } \\
\hline Senna bicapsularis (L.) Roxb. & $A$ & $\mathrm{EE}$ & & & & & & & 5.9 & & 3.9 & & & & 1.72 \\
\hline \multicolumn{16}{|l|}{ Combretaceae } \\
\hline \multicolumn{16}{|l|}{ Convolvulaceae } \\
\hline Ipomoea jalapa (L.) Pursh & B & $\mathrm{EE}$ & & & & & & & & & & & & 2.9 & 1.04 \\
\hline Ipomoea microsepala Benth. & B & $\mathrm{EE}$ & & & & & & & & & & & & 3.7 & 1.13 \\
\hline Ipomoea squamosa Choisy & B & $\mathrm{EE}$ & & & & & & & & & 5.6 & & 3 & 10.3 & 3.31 \\
\hline Ipomoea tiliacea (Willd.) Choisy & B & $\mathrm{EE}$ & & & & 3.7 & & & & & & & & & 2.15 \\
\hline Ipomoea sp. 1 & B & $\mathrm{EE}$ & 9.7 & & & & & & & & & & & & 2.34 \\
\hline Ipomoea sp. 2 & B & $\mathrm{EE}$ & 7.9 & & 5.7 & & & & & & & & & & 1.81 \\
\hline \multicolumn{16}{|l|}{ Cucurbitaceae } \\
\hline Cionosicys macranthus (Pittier) C. Jeffrey & B & $\mathrm{EE}$ & 4.1 & & & & & & & & & & & & 0.82 \\
\hline Cucumis anguria $\mathrm{L}$. & B & $\mathrm{EE}$ & & & & & & & & & 1.2 & & & & 0.75 \\
\hline
\end{tabular}


Apéndice 1. Continuación

\begin{tabular}{|c|c|c|c|c|c|c|c|c|c|c|c|c|c|c|c|}
\hline & FB & F Crec & $\mathrm{CF}$ & ED & $\begin{array}{l}\text { LG- } \\
\mathrm{Ch}\end{array}$ & LM & Apo & Man & RBp & RBt & $\mathbf{R L}$ & Con & Som & Pop & $\begin{array}{l}\text { VIR } \\
\text { global }\end{array}$ \\
\hline Cucurbita argyrosperma K. Koch & B & $\mathrm{EE}$ & 2.4 & & & & & & & & & & & & 0.95 \\
\hline \multicolumn{16}{|l|}{ Euphorbiaceae } \\
\hline Phyllanthus fluitans Benth. ex Müll. Arg. & $\mathrm{H}$ & LF & & & & & & & & & 2.6 & & & & 0.88 \\
\hline Tragia volubilis L. & $\mathrm{B}$ & $\mathrm{EE}$ & & & & & & & & & 0.9 & & & & 0.69 \\
\hline \multicolumn{16}{|l|}{ Fabaceae } \\
\hline Aeschynomene deamii B.L. Rob. \& Bartlett & $\mathrm{H}$ & $\mathrm{EE}$ & 2.4 & & & & & & & & 2.6 & 2.1 & 2.6 & & 1.82 \\
\hline Aeschynomene scabra G. Don. & $\mathrm{H}$ & $\mathrm{EE}$ & & & & & & & & & & 5.2 & & & 1.37 \\
\hline Dalbergia brownei (Jacq.) Schinz & $\operatorname{Ar}$ & $\mathrm{EE}$ & & & & 4.7 & & & 7 & & & 1.8 & & & 3.39 \\
\hline Machaerium falciforme Rudd. & $\mathrm{B}$ & $\mathrm{EE}$ & & & & & & & & & & 1.2 & & & 0.78 \\
\hline Sesbania sp. & B & $\mathrm{EE}$ & & & & 5 & & & & & & 1.2 & & & 2.29 \\
\hline Vigna luteola (Jacq.) Benth. * & B & $\mathrm{EE}$ & 14 & & & & & & & & 1.7 & 1.8 & 2.6 & & 7.61 \\
\hline \multicolumn{16}{|l|}{ Hippocrateaceae } \\
\hline Hippocratea celastroides Kunth & $\mathrm{Ar}$ & $\mathrm{EE}$ & 3 & & & & & & & & & & & & 1.28 \\
\hline Hippocratea volubilis L. & $\mathrm{Ar}$ & $\mathrm{EE}$ & & & & & & & 2.9 & & & & & & 0.9 \\
\hline \multicolumn{16}{|l|}{ Lamiaceae } \\
\hline Salvia sp. & $\mathrm{H}$ & $\mathrm{EE}$ & 5.8 & & & 7.2 & & & & & & & & & 3.72 \\
\hline \multicolumn{16}{|l|}{ Malvaceae } \\
\hline $\begin{array}{l}\text { Kosteletzkya depressa (L.) O.J. Blanch., } \\
\text { Fryxell \& D.M. Bates }\end{array}$ & $\mathrm{H}$ & $\mathrm{EE}$ & & & & & & & & & 1.2 & & & & 0.75 \\
\hline $\begin{array}{l}\text { Malvaviscus arboreus Cav. } \\
\text { Mimosaceae }\end{array}$ & \multicolumn{14}{|c|}{ Mimosaceae } & 2.94 \\
\hline Acacia cornigera (L.) Willd. & $\mathrm{Ar}$ & $\mathrm{EE}$ & & & & & & 6 & 7 & & & & & & 1.43 \\
\hline Mimosa pigra L. & $\mathrm{Ar}$ & $\mathrm{EE}$ & & & & & & & & & 5.6 & & & 7.5 & 2.67 \\
\hline Mimosa sp. & $\mathrm{H}$ & $\mathrm{EE}$ & & & & & 3.9 & 8.2 & & & & & & & 1.02 \\
\hline $\begin{array}{l}\text { Pithecellobium insigne Micheli } \\
\text { ex Donn. Sm. }\end{array}$ & A & $\mathrm{EE}$ & & & & & & & 18.3 & & & & & & 2.33 \\
\hline Zygia recordii Britton \& Rose & A & $\mathrm{EE}$ & & & & 1.8 & & & & & & & & & 1.29 \\
\hline \multicolumn{16}{|l|}{ Moraceae } \\
\hline Ficus aurea Nutt. & A & $\mathrm{EE}$ & & & & & & & 6.3 & & & & & & 1.06 \\
\hline \multicolumn{16}{|l|}{ Nymphaeaceae } \\
\hline Nymphaea conardii Wiersema & $\mathrm{H}$ & $\mathrm{EHF}$ & & & & & & & & & & 1.2 & & & 0.78 \\
\hline Nymphaea pulchella DC. & $\mathrm{H}$ & EHF & & & & & & & 2.9 & & & & & & 0.8 \\
\hline \multicolumn{16}{|l|}{ Onagraceae } \\
\hline Ludwigia adscendens (L.) H. Hara & $\mathrm{H}$ & $\mathrm{EE}$ & & & & & & & & & & 0.6 & & & 0.69 \\
\hline Ludwigia octovalvis (Jacq.) P.H. Raven & $\mathrm{H}$ & $\mathrm{EE}$ & & & & & & & & & 3.7 & 0.6 & & & 1.12 \\
\hline \multicolumn{16}{|l|}{ Passifloraceae } \\
\hline Passiflora filipes Benth. & $\mathrm{B}$ & $\mathrm{EE}$ & & & & & 3.9 & & & & & & & & 0.74 \\
\hline Passiflora foetida L. & $\mathrm{B}$ & $\mathrm{EE}$ & & & & & & & & & 1.7 & 1.2 & & & 0.97 \\
\hline Passiflora sp. & B & $\mathrm{EE}$ & & & & 0.5 & & & & & & & & & 0.74 \\
\hline \multicolumn{16}{|l|}{ Plantaginaceae } \\
\hline Bacopa monnieri (L.) Wettst. & $\mathrm{H}$ & $\mathrm{EE}$ & & & & & & & & 10.1 & 1.2 & 2.6 & & & 1.93 \\
\hline \multicolumn{16}{|l|}{ Polygonaceae } \\
\hline Polygonum longiocreatum Bartlett & $\mathrm{H}$ & $\mathrm{EE}$ & & & & & & & & & & & & 2.9 & 1.04 \\
\hline \multicolumn{16}{|l|}{ Rubiaceae } \\
\hline Randia laetevirens Standl. & $\mathrm{Ar}$ & $\mathrm{EE}$ & & & & & & & 2.9 & & & 2.4 & & & 1.22 \\
\hline \multicolumn{16}{|l|}{ Rutaceae } \\
\hline Zanthoxylum caribaeum Lam. & $\mathrm{Ar}$ & $\mathrm{EE}$ & & & & 7.6 & & & 2 & & & & & & 3.41 \\
\hline
\end{tabular}


Apéndice 1. Continuación

FB F Crec CF $\begin{array}{cccccccccc}\text { LG- } & \text { LM } & \text { Apo Man } & \text { RBp RBt } & \text { RL Con Som Pop } \begin{array}{c}\text { VIR } \\ \text { global }\end{array}\end{array}$

\section{Sapindaceae}

Paullinia costaricensis Radlk.

Solanaceae

Physalis arborescens L.

Solanum tampicense Dunal

Umbelliferae

Hydrocotyle bonariensis Lam.

Urticaceae

Boehmeria cylindrica (L.) Sw.

Verbenaceae

Lantana camara L.

Lippia dulcis Trevir.

Lippia nodiflora (L.) Michx.

Vitaceae

Cissus sicyoides L.

B $E E$

H EE

Ar EE

H EE

H $\quad$ EE 16

Ar EE

H $\quad$ EE 3.7

H EE

B $\quad E E$

(n)

2.9

0.84

2

4.1

$2.9 \quad 1.2$

0.78

1.5

$9.6 \quad 11.1$

$\begin{array}{lll}4.6 & 1.2 & 5.5\end{array}$

2.98

3.37

6

0.81

1.32

0.6

$1.2 \quad 2.6$

0.91 
Apéndice 2. Descripción de las agrupaciones florísticas que se formaron en el dendrograma presentado en la figura 4

Agrupación 1: Typha domingensis (14 muestras)

Localización: Laguna Grande y Chica, Río Limón, aunque predomina en La Mancha.

Riqueza: media de seis especies y un máximo de 10 especies por cuadro.

Especies frecuentes asociadas: S. lancifolia subsp. media (fundamentalmente en La Mancha), Z. miliacea (Río Limón y La Mancha), Z. caribaeum (La Mancha). Otras especies menos frecuentes son Scleria sp., Cynodon dactylon y Salvia sp.

Suelos e hidroperiodo: histosol sáprico principalmente en la región centro-norte y sobre histosol fíbrico en el Papaloapan, con inundación moderada a prolongada. Solo hay una muestra sobre gleysol hístico en Laguna Grande y Chica.

Agrupación 2: Sagittaria lancifolia subsp. media- Typha domingensis (8 muestras)

Localización: solamente en La Mancha.

Riqueza: cuatro especies por cuadro y un valor máximo de cinco especies por cuadro.

Especies frecuentes asociadas: Scleria sp. y Sesbania sp.

Suelos e hidroperiodo: histosol fíbrico con inundación prolongada.

Agrupación 3: Echinochloa pyramidalis y T. domingensis (14 muestras)

Localización: solamente en La Mancha.

Riqueza: media de dos especies y un máximo de cinco especies por cuadro.

Especies frecuentes asociadas: Dalbergia brownei, Ipomoea tiliacea. El pasto alemán, E. pyramidalis, puede Ilegar a ser monodominante y excluir a las otras especies.

Suelos e hidroperiodo: histosol sáprico con inundación baja a moderada.

Agrupación 4: Cyperus giganteus y Thalia geniculata (26 muestras)

Localización: 4 sitios.

Riqueza: media de cinco, con un valor máximo de especies en un cuadro de 14.

Especies frecuentes asociadas: en la zona norte en Estero Dulce dominan T. geniculata y C. giganteus con menor frecuencia y Echinochloa pyramidalis aparece en algunas muestras. En Laguna Grande-Laguna Chica se presenta una dominancia de C. giganteus y T. domingensis. En la zona del Papaloapan el humedal de Sombrerete presenta además de las especies dominantes a Pontederia sagitatta y Limnocharis flava; por su parte La Popotera es considerablemente más rico en especies: Hymenachne amplexicaulis, Thalia geniculata, Mimosa pigra, Scleria sp., Sagittaria latifolia y la palma Attalea butyracea.

Suelos e hidroperiodo: histosol fíbrico con inundación moderada a prolongada en el Papaloapan, y sobre gleysol mólico con inundación baja y moderada en dos sitios de la zona centro norte.

Agrupación 5: Leersia sp.-Typha domingensis (7 muestras)

Localización: Estero Dulce, Laguna Apompal y Laguna Grande-Laguna Chica.

Riqueza: media de tres y máximo de cinco.

Especies frecuentes asociadas: Pontederia sagittata, Sagittaria lancifolia, Hydrocotyle bonariensis.

Suelos e hidroperiodo: gleysol hístico y mólico con inundación baja a moderada e inundación prolongada (2 muestras Laguna Grande -Chica y una muestra en Estero Dulce respectivamente) y sobre histosol fíbrico con inundación prolongada (Apompal).

Agrupación 6: Leersia ligularis, Cyperus articulatus, Sagittaria lancifolia, Pontederia sagittata (17 cuadros)

Localización: Laguna Apompal, Laguna Mandinga, Río Blanco, Río Limón, Laguna Conejo. Riqueza: media de especies por cuadro de 10 y el número máximo de 21.

Especies frecuentes asociadas: Hydrocotyle bonariensis, Eleocharis cellulosa, Sagittaria lancifolia, Pontederia sagittata, Laguncularia racemosa (solamente en Laguna Conejo).

Suelos e hidroperiodo: predomina sobre suelos gleysol hístico con inundación moderada y en un caso con salinidades más altas (gleysol hístico endosálico en Río Blanco), así como sobre histosol tiónico con inundación baja, y sobre histosol fíbrico con inundación moderada, todos ellos en el Papaloapan. Hay una sola muestra en la zona centro norte sobre histosol fíbrico con inundación prolongada (una muestra en Apompal).

Agrupación 7: Paspalum sp., Eleocharis cellulosa y Bacopa monnieri (4 muestras) Localización: tular de Río Blanco.

Riqueza: media de seis y el máximo es ocho.

Especies frecuentes asociadas: Fimbristylis spadicea.

Suelos e hidroperiodo: gleysol hístico endosálico con inundación prolongada. 
Apéndice 2. Continuación

Agrupación 8: Pithecellobium calostachys- Spartina spartinae (4 muestras)

Localización: popal de Río Blanco.

Riqueza: media de 12 y el valor máximo de 18.

Especies frecuentes asociadas: Malvaviscus arboreus, Hymenachne amplexicaulis, Leersia ligularis, Sabal mexicana (ocasional). Suelos e hidroperiodo: histosol sáprico con inundación prolongada.

Agrupación 9: Boehmeria cylindrica, Vigna luteola, Luziola peruviana y Setaria palmifolia (10 muestras).

Localización: Ciénaga del Fuerte.

Riqueza: media de seis especies y máximo de ocho.

Especies frecuentes asociadas: Thalia geniculata, Setaria paniculifera, y dos especies de Ipomoea sin identificar.

Suelos e hidroperiodo: gleysol mólico con inundación moderada. 